\title{
Marco propositivo de relaciones entre innovación colaborativa abierta y comunicación estratégica: aplicación a un grupo de empresas de origen antioqueño*
}

\section{Ángela Preciado-Hoyos ${ }^{1}$}

Recibido: 26/02/2019

Aprobado: 21/06/2019
Enviado: 03/03/2019

Aceptado: 21/06/2019

DOI: 10.5294/pacla.2020.23.2.6

\section{Para citar este artículo / to reference this article / para citar este artigo}

Preciado-Hoyos, A. (2020). Marco propositivo de relaciones entre innovación colaborativa abierta y comunicación estratégica: aplicación a un grupo de empresas de origen antioqueño. Palabra Clave, 23(2), e2326. https://doi.org/10.5294/pacla.2020.23.2.6

\section{Resumen}

En este artículo se presenta un marco propositivo sobre los elementos que entran en relación en un proceso de innovación colaborativa abierta con grupos de interés, visto desde una perspectiva estratégica de la comunicación. La verificación del marco propuesto se hizo en un grupo de ocho empresas que tienen su sede principal en la región de Antioquia, cotizan en la Bolsa de Valores de Colombia y operan en diferentes países de América Latina. La metodología consistió en entrevistas en profundidad, basadas en preguntas semiestructuradas. Los resultados muestran una presencia significativa de los elementos identificados en el marco propuesto, en las actividades de innovación de las organizaciones de la muestra y en las áreas que se ocupan del proceso y el acompañamiento que hacen los encargados

\footnotetext{
La investigación que aquí se reporta hace parte de una más amplia titulada "Innovación colaborativa abierta y comunicación mediada por TIC en las empresas: un análisis desde las culturas latinoamericanas”, realizada en el Centro de Investigaciones de la Comunicación Corporativa y Organizacional (Cicco), de la Universidad de La Sabana, financiada con recursos de la Dirección de Investigación de la misma universidad. Código COM-72-2016.
}

$1 \bowtie$ https://orcid.org/0000-0002-0420-5471. Universidad de La Sabana, Colombia. angela.preciado@unisabana.edu.co 
de la comunicación en la construcción de una cultura de la innovación. Se concluye que deberá incrementarse la participación de los profesionales de la comunicación en los procesos de innovación.

\section{Palabras clave (Fuente: tesauro de la Unesco)}

Innovación colaborativa abierta; grupos de interés; comunicación estratégica; innovación; empresas; Colombia. 


\section{Purposive Framework of Relations between Open Collaborative Innovation and Strategic Communication: Application to a Group of Businesses from Antioquia*}

\section{Abstract}

This article introduces, from a strategic communication perspective, a purposive framework of elements that relate to each other in an open collaborative innovation process with stakeholders. The proposed framework was verified with a group of eight companies whose principal place of business is in Antioquia, are listed on the Colombian Stock Exchange, and operate in different Latin American countries. The method consisted of in-depth interviews with semi-structured questions. Results show that the elements identified in the proposed framework are significantly present in the sampled organizations' innovation activities, the areas that oversee the process, and the support provided by communicators in building a culture of innovation. In conclusion, communication experts' involvement in innovation should increase.

\section{Keywords (Source: Unesco Thesaurus)}

Open collaborative innovation; interest groups; strategic communication; innovation; enterprises; Colombia.

\footnotetext{
* The research reported herein is part of a broader one entitled "Open Collaborative Innovation and ICT-Mediated Communication in Companies: An Analysis from Latin American Cultures” conducted by the Center for Corporate and Organizational Communication Research (Cicco), Universidad de La Sabana, and financed with resources from the Research Department at the same university. Code: COM-72-2016.
} 


\section{Enquadramento propositivo de relações entre inovação colaborativa aberta e comunicação estratégica: aplicação a um grupo de empresas de Antioquia, Colômbia*}

\section{Resumo}

Neste artigo, é apresentado um enquadramento propositivo sobre os elementos que entram em relação em um processo de inovação colaborativa aberta com grupos de interesse, visto de uma perspectiva estratégica da comunicação. A verificação do enquadramento proposto foi feita em um grupo de oito empresas com sede principal em Antioquia, que cotizam na Bolsa de Valores da Colômbia e operam em diferentes países da América Latina. A metodologia consistiu em entrevistas em profundidade, baseadas em perguntas semiestruturadas. Os resultados mostram uma presença significativa dos elementos identificados no enquadramento proposto, nas atividades de inovação das organizações da amostra e nas áreas que tratam do processo e do acompanhamento que os encarregados da comunicação fazem na construção de uma cultura da inovação. Conclui-se que a participação dos profissionais da comunicação deve aumentar nos processos de inovação.

\section{Palavras-chave (Fonte: tesauro da Unesco)}

Inovação colaborativa aberta; grupos de interesse; comunicação estratégica; inovação; empresas; Colômbia.

Esta pesquisa faz parte de uma mais ampla intitulada "Innovación colaborativa abierta y comunicación mediada por TIC en las empresas: un análisis desde las culturas latinoamericanas" ("Inovação colaborativa aberta e comunicação mediada pelas TIC nas empresas: uma análise a partir das culturas latino-americanas”), realizada no Centro de Pesquisas da Comunicação Corporativa e Organizacional da Universidad de La Sabana, com o apoio financeiro de recursos da Direção de Pesquisas da mesma universidade. Código COM-72-2016. 


\section{Introducción}

Muchas organizaciones del mundo están implementando la innovación colaborativa abierta (ICA) en sus procesos. Algunos trabajos realizados en otras latitudes dan cuenta de prácticas de comunicación en programas de apoyo a la innovación abierta de las organizaciones (Patsch \& Zerfass, 2013; Tossavainen, Shiramatsu, Ozono \& Shintani, 2016). Aunque Colombia es un país que cuenta con diferentes estamentos públicos y privados para apoyar la innovación de las empresas, ${ }^{2}$ una búsqueda realizada en marzo de 2017 de investigaciones que relacionaran la comunicación con procesos de innovación organizacional, publicados en los últimos diez años en revistas académicas indexadas en ISI Web of Knowledge y en Scopus, mostró que no existían estudios sobre esta temática concreta, centrados en este país de América Latina.

Colombia ocupó el puesto 63 en el Global Innovation Index 2018 (Dutta, Lanvin \& Wunsch-Vincent, 2018). ${ }^{3}$ En América Latina, en este mismo listado, le anteceden Chile, que es el primer país de la región que aparece (puesto 47), Costa Rica, México y Uruguay. En este índice, Colombia está por encima de países más grandes y con economías que se han caracterizado por ser más robustas, como Brasil. En el ranking de innovación desarrollado y aplicado en Colombia por la Asociación Nacional de Empresarios de Colombia (ANDI) y la Revista Dinero, publicado en $2018,{ }^{4}$ se reconocen algunas fortalezas que tiene el país en la materia, aunque también se reportan debilidades.

En cuanto a las fortalezas, están la mejora en la sofisticación de los mercados y las empresas, la facilidad para acceder a créditos, la proporción

2 Algunas de estas entidades son Colciencias, Renata, Cultura E, Colombia Emprende, Innpulsa, Consejo Privado de Competitividad Nacional, Consejo Nacional de la Economía Naranja, Ruta N.

3 Este ranking es elaborado por Cornell SC Johnson College of Business, Instead y World Intelectual Property Organization (WIPO). Se aplica en 126 economías que representan el 90,8 \% de la población mundial y el 96,3\% del PIB del planeta. Este índice utiliza 80 indicadores para hacer la medición. Además de las medidas clásicas de innovación, incluye otras como el nivel de desarrollo, el entorno político, la educación, la infraestructura, la sofisticación empresarial y la investigación de cada país.

4 Este ranking mide cuatro dimensiones: a) condiciones o factores que deben existir en las organizaciones para que la innovación sea exitosa, con un peso del $10 \%$ en la medición; b) capacidades, que son la forma en que la empresa genera y transforma nuevo conocimiento para aprovechar oportunidades y solucionar retos estratégicos, con un peso del $40 \%$; y c) resultados, entendidos como la materialización de innovaciones de producto, proceso y comercialización en el mercado, con un peso del $50 \%$ de la medición (Dinero, 2018). 
de organizaciones privadas que brinda capacitación formal a sus empleados, la producción limpia de muchas compañías locales y el número de compañías certificadas en calidad con la norma ISO 14001:2015. Las principales desventajas son la inestabilidad política y la situación de orden público, así como un bajo nivel de joint ventures o alianzas estratégicas entre empresas y el alto nivel de sus aranceles, el cual ha ido bajando gracias a los tratados de libre comercio.

En aspectos como el orden público, se esperan cambios positivos debido al acuerdo de paz firmado en 2016 entre el Gobierno de Juan Manuel Santos y las Fuerzas Armadas Revolucionarias de Colombia (FARC). En cuanto a los esfuerzos del Gobierno actual, del presidente Iván Duque, en materia de impulso a la innovación, destacan el apoyo al crecimiento de las industrias creativas, en leyes que promueven la economía naranja, como la Ley 1834/2017, de 23 de mayo, y la creación del Ministerio de Ciencia, Tecnología e Innovación (Minciencias), aprobada por el mismo estamento en diciembre de 2018 y en espera de convertirse en ley.

Es decir, aunque existen varios aspectos que deben mejorar, identificados por entes como el Consejo Privado de Competitividad (CPC), ${ }^{5}$ el Departamento Administrativo de Ciencia, Tecnología e Innovación (Colciencias) ${ }^{6} \mathrm{o}$ la propia ANDI, este trabajo se orienta a determinar los asuntos en los que se puede incidir desde la comunicación, en lo que atañe a los elementos de la organización y su entorno, que entran a desempeñar un papel protagonista cuando se busca establecer y fortalecer relaciones estratégicas con grupos de interés, como se contempla en la implementación de modelos de ICA. Estos últimos vienen imponiéndose como consecuencia de las posibilidades que han generado los desarrollos tecnológicos, la globalización y las nuevas prácticas para compartir el conocimiento, las cuales, como aquí se expone, se soportan en buena medida en la comunicación para materializarse.

5 "El Consejo Privado de Competitividad Nacional es una organización sin ánimo de lucro cuyo objeto es contribuir de manera directa en la articulación de estrategias que, en el corto, mediano y largo plazo, permitan lograr mejoras significativas en el nivel de competitividad de Colombia" (CPC, 2019).

6 Colciencias es la entidad estatal encargada de promover las políticas públicas para fomentar la ciencia, la tecnología y la innovación en Colombia. 


\section{De la innovación a la ICA}

Innovar es quizá la manera más valorada de competir en los negocios y de solucionar problemas de diversa índole. Una empresa innovadora es aquella que tiene una estrategia para desarrollar e introducir nuevos productos o servicios antes que sus competidores, pero se caracteriza, sobre todo, por que las nuevas aplicaciones son útiles y bien recibidas por los mercados.

En 1934, Joseph Alois Schumpeter señaló que existían dos maneras de conseguir avances tecnológicos: inventar e innovar. La primera se asocia, por lo general, con cambios disruptivos, en tanto que la segunda suele relacionarse más con aplicar transformaciones de manera incremental. En este trabajo, se considera que inventar algo totalmente nuevo puede considerarse una innovación radical. Y aunque suele establecerse una relación más estrecha entre innovación y desarrollo de tecnologías informáticas, o entre innovación y robótica, la innovación también tiene lugar en procesos organizativos que pueden abarcar la prestación de servicios, los ajustes a las estructuras de las entidades, la distribución de productos o la innovación social, orientada a mejorar aspectos como la comunicación, el bienestar de las personas o la prestación de un servicio público (Hartley, Sørensen \& Torfing, 2013).

Durante muchos años las preguntas de las compañías y de las sociedades en torno a la innovación han sido cómo mejorar las condiciones para conseguirla y cómo hacer énfasis en innovaciones que aporten mayor diferencia en un mercado. Así, en el pasado, las empresas concebían la innovación como una fortaleza que se generaba de puertas para dentro. En aquel contexto, quienes buscaban competir con los líderes de un sector tenían que disponer de sus propios recursos para crear laboratorios si querían tener alguna oportunidad, y las exploraciones en investigación y desarrollo se hacían de manera secreta, de modo que no fueran copiadas por los competidores (Chesbrough, 2007).

El escenario cambió debido a diversas circunstancias. Por una parte, las empresas empezaron a enfrentar dificultades derivadas de la alta segmentación de los mercados, entre ellas, la demanda de una arquitectura diferente 
para cada tipo de producto. A ello se sumaron procesos como la globalización y el acelerado desarrollo de las tecnologías de la información y de la comunicación (TIC). Este, a la vez que introdujo nuevas demandas, multiplicó las posibilidades de democratizar y compartir el conocimiento (Chesbrough, 2004), así como una mayor valoración del capital intelectual, que ha servido de motor para impulsar nuevos modelos de innovación, entre ellos, la innovación abierta. Esta última se define como el uso de entradas y salidas intencionales de conocimiento para acelerar la innovación interna y expandir los mercados para el uso externo de la innovación, respectivamente (Chesbrough \& Crowther, 2006). Se da cuando las compañías buscan comercializar sus propias ideas, pero también las iniciativas de otras empresas, o cuando combinan estas dos maneras de obtener ideas o de ponerlas a funcionar en los mercados (Gassman \& Enkel, 2004). Ello lo hacen desde la premisa de que el conocimiento está en todas partes, se puede utilizar para aplicar al propio negocio u obtener utilidades por la explotación de esos nuevos conocimientos en negocios ajenos. Es, por consiguiente, una perspectiva que saca provecho de la sociedad del conocimiento, por cuanto valora el capital intelectual que circunda el ejercicio empresarial (Chesbrough, 2004; West \& Gallager, 2006). Otras ventajas del modelo abierto vinculan ciclos más cortos para innovar y menor inversión en costos, si se compara con los paradigmas cerrados, pues, más que instalar estructuras, es acercar a la empresa el conocimiento que existe en el entorno.

Estudios posteriores a los realizados en la primera década del siglo XXI agregan otras ventajas, tales como alcanzar colaboración en procesos que son más interactivos, de los que también pueden sacar provecho las partes que intervienen (Piller \& West, 2013), o ganar tiempo, al no tener que empezar de cero cada nueva iniciativa, porque es posible construir sobre un saber previo, que está disponible (Conrado, Karlsson, Romero, Sarr \& Wilkins, 2017).

$\mathrm{Al}$ integrar el concepto de colaboración en la IC, se complementan la voluntad y los conocimientos de unos y otros (Ferdinand \& Meyer, 2017), lo que obliga a conectarse e integrarse en un amplio rango de actividades y habilita a los responsables de estos procesos a repensar el diseño de estra- 
tegias colaborativas en un mundo que funciona en red (Lin, 2017). La ICA surgió como consecuencia de estos desarrollos, sobre todo, por un mayor acceso a internet y, en general, a las TIC. Posibilita crear un valor organizacional compartido y se propone que las ventajas y ganancias sean apropiadas por empresa y grupos de interés ${ }^{7}$ en igualdad de condiciones, siempre en todas las etapas del proceso, para que se esté atendiendo a un verdadero concepto de apertura (Lee, Olson \& Trimi, 2012; Islam, Miller \& Park, 2017). En la ICA cuentan el número y la procedencia de las fuentes que aportan y el interés en compartir las ideas que surgen dentro de la organización, con otras de fuera o a la inversa (Baldwin \& Von Hippel, 2011). Un aspecto interesante de este modelo es que la organización se convierte en punto de encuentro, contacto o confluencia de diversas personas que se suman para pensar en soluciones a un problema. Un caso concreto fue el desarrollo cooperativo de diseñadores y programadores independientes, quienes crearon nuevas aplicaciones que luego fueron usadas en diversos productos informáticos (Gassman \& Enkel, 2004).

De acuerdo con lo anterior, las ideas pueden surgir dentro de las organizaciones, pero también más allá de sus límites. Esa suerte de "barreras", que en la literatura sobre comunicación de las empresas y en la relativa a la innovación discriminaban entre lo interno y lo externo, se han convertido en membranas semiimpermeables que facilitan que las iniciativas transiten de manera fluida (West \& Bogers, 2017). El incremento en el uso de las TIC por parte de las organizaciones ha contribuido a los procesos de innovación y ha repercutido en nuevas formas de organizarse. Gracias a ellas, innovar no tendría por qué ser tan costoso, porque, sin necesidad de echar mano de grandes estructuras, acrecentaron el trabajo en red y permitieron que se incrementaran los intercambios de ideas. La rapidez y apertura que posibilitan estos medios da cabida a una mayor participación de personas, lo que garantizaría que los productos o servicios se ajusten mejor a las necesidades de los usuarios.

Para Dahlander y Gann (2010) el punto de partida de la idea de apertura es que la organización esté articulada con diferentes tipos de socios para

7 En este artículo se utilizan como sinónimos las denominaciones grupos de interés y stakeholders. 
adquirir ideas y recursos de ellos. Puede ser entendida, asimismo, como la manera en que las firmas adquieren experiencia desde el exterior, trabajan para crear sinergia entre sus propios procesos y los que están disponibles por fuera, de manera que pueden ser capaces de crear beneficios, productos y servicios que tienen acogida.

Las redes de relaciones desempeñan un papel central en la creación y distribución del conocimiento en muchos campos (Dittrich \& Duysters, 2007; Powell, 1990). Al respecto, Von Hippel (1988) identificó cuatro fuentes externas de innovación para las compañías, las cuales son equiparables con los grupos de interés de estas: a) proveedores y clientes, b) universidades, Gobierno, centros de investigación y laboratorios privados, c) competidores y d) otras naciones. El aporte de estos grupos puede darse de muchas maneras y obedecer a distintos niveles de compromiso, por una parte, o de compensación, por otra. Las investigaciones empíricas han demostrado el impacto que tiene integrar a los clientes, y a otros stakeholders, en los resultados de las compañías en este tipo de programas (Ingenbleek \& Dentoni, 2016).

Si como se ha dicho la ICA habilita a la organización para que sea más efectiva en la creación y en la captura de valor porque ayuda a obtener un mayor número de ideas debido a la inclusión de un amplio número de agentes y conceptos externos utilizando las TIC, también ayudan la posición y el reconocimiento que tenga la empresa para atraer ideas de fuentes diversas (Chesbrough, 2007), o, como se expone aquí, puede crear valor a partir de las relaciones que establece y mantiene con los grupos y con las personas que gravitan en sus entornos (Dawson, Young, Tu \& Chongyi, 2014). En este intercambio, la comunicación es la que habilita los nexos o fortalece los que ya se tienen.

\section{La comunicación estratégica en los procesos de innovación}

La calidad de la relación que tiene la organización con sus grupos de interés se convierte en fuente de ventaja competitiva y de creación de valor, pues, cuanto mejor conectada esté, tendrá muchas más oportunidades para 
desarrollar la ICA (Lee, Olson \& Trimi, 2012). Dahlander y Gann (2010) destacan al respecto que las firmas varían sus formas de innovar, de acuerdo con el grado en el que detectan, evalúan y asimilan inputs externos y se acomodan a sus entornos para convivir con ellos.

No cabe duda de que muchos proveedores o clientes tienen iniciativas y conocimientos que pueden servir a las compañías (Carillo, Huff \& Chawner, 2017), generan contenidos, son prosumidores y, en muchos casos, se comportan como profesionales aunque en la ICA no existe restricción acerca del tipo de colaboradores con los que se puede trabajar (Mahr \& Lievens, 2012; Marchi, Giachetti \& de Gennaro, 2011; Pahalad \& Ramaswamy, 2000; Sagaris, 2014). En estudios sobre estas cuestiones, se han establecido tipologías, entre las cuales se distingue entre usuarios comunes, usuarios profesionales y usuarios líderes (Füller, Matzler, Hutter \& Hautz, 2012), y se proponen algunas cualidades asociadas al dominio específico de habilidades, capacidad creativa y motivaciones para participar en actividades de innovación colaborativa (Wakefield \& Wakefield, 2016).

Para comprender el aporte que puede hacer la comunicación en los procesos de ICA, conviene ubicarse en un enfoque de comunicación estratégica, por cuanto este propone una visión dialógica y de reflejo entre la organización y su entorno. Esta visión, que en castellano se puede traducir como reflexiva, por provenir de la expresión inglesa reflective, se refiere a la manera como el entorno se expresa o se manifiesta en las acciones y en los comportamientos de la organización (Verhoeven, Zerfass \& Tench, 2011). El punto de partida no es ver a esta última como un ente aislado de las situaciones sociales, sino como parte de ellas y con un poder de transformar e incidir en la cultura, por la vía de la comunicación. Ahora bien, se trata de un poder que comparte en igual proporción con otros agentes de la esfera pública.

Esta perspectiva tiene pertinencia ahora, más que en el pasado, porque las organizaciones, sin importar el sector del que se trate, han dejado de depender de los medios periodísticos para divulgar sus puntos de vista, disponen de medios propios de comunicación y equipos de profesionales 
capacitados para dar visibilidad a sus valores e intereses (Savič, 2016). Luego, si conquistan la legitimidad de sus actuaciones, terminan siendo consideradas parte de la sociedad (Verhoeven et al., 2011). El carácter de reflejo se materializa, entre otras circunstancias, cuando los profesionales de la comunicación analizan los cambios en normas, valores y asuntos sociales que se dan en el entorno y luego aconsejan a los cuadros directivos sobre cómo se debe interactuar en él.

Desde el campo de estudios de la comunicación, la naturaleza innovadora de las empresas ha sido reconocida como una de las variables con más peso cuando se mide la reputación corporativa (Fombrun, 1996; Safón, 2009), pues una compañía innovadora se destaca por su predisposición hacia el aprendizaje y por su mentalidad orientada a experimentar para optimizar productos, servicios o procesos; estimula la competencia interna y la tolerancia al fracaso; promueve a los líderes creativos; y trabaja de manera incesante para renovar el portafolio de ofertas (Kratzer, Meissner \& Roud, 2017).

En igual sentido, es pertinente afirmar que en el área de comunicaciones reside, quizá más que en ninguna otra, la responsabilidad de escuchar el entorno, para ajustarse a las expectativas sociales, por cuanto la gestión de las relaciones está en cabeza del profesional de la comunicación (Bowen, 2008). Esta mirada contrasta con la visión instrumental y de mera difusión de informaciones, que suele instalarse en el medio latinoamericano y que se atribuye, por lo general, a las escuelas de estudio de la comunicación en las organizaciones que surgieron en Estados Unidos en la era industrial (Holtzhausen \& Zerfass, 2015).

A la función del profesional de la comunicación como agente que trabaja en los límites de la organización, para conectarla con su entorno, se le ha denominado, en inglés, boundary spanning. Dicha función tiene dos partes: a) el desarrollo de actividades outbound, mediante las cuales los profesionales facilitan las actividades del negocio y construyen asuntos que la organización aporta a la sociedad, y b) actividades inbound, con las cuales se aseguran de que la organización pueda maniobrar en la esfera pública y ajustar las estrategias corporativas a las demandas de aquella. Estas últimas 
actividades tienden a ser más reflexivas y, por tanto, más estratégicas. Se ha encontrado, no obstante, que las actividades outbound son desarrolladas más frecuentemente que las actividades inbound (Verhoeven et al., 2011).

Si los públicos de la organización dominan el uso de medios sociales y tienen acceso a ellos, e incluso si han tenido la capacidad de movilizar a otros ciudadanos en torno a algunas causas para ejercer presión en las compañías, ha sido, en buena medida, debido al acceso que tienen a las TIC. De ahí que hoy existe un contenido más complejo en la tarea del profesional del área, que se integra en la esencia de sus funciones en el momento de apoyar los procesos de la ICA. De este modo, si se retoman las tareas del comunicador como agente estratégico, él puede aportar a la innovación desde diversos frentes, entre ellos:

- Motivando y convocando a diferentes actores en torno a los proyectos de la organización para combinar ideas de cambio y proponer soluciones técnicas.

- Vislumbrando dónde se pueden crear oportunidades de trabajo colaborativo y, en ese sentido, apoyar la creación de comunidades o "ecosistemas de innovación” con los cuales se pueda relacionar la compañía (Ferdinand \& Meyer, 2017).

- Generando y fortaleciendo un sistema de comunicación orientado a escuchar a los grupos de interés (Macnamara, 2015).

- Acompañando las etapas que abarca la innovación: adquisición, asimilación, transformación y explotación. En estas fases, se pueden presentar tensiones en lo que atañe a las relaciones entre los actores que participan en ellas, por lo que se puede trabajar en torno a esquemas de negociación y convivencia (Ingenbleek \& Dentoni, 2016; Hoes, Beers \& Van Mierlo, 2016).

- Aprovechando las TIC, gracias a las cuales es posible comprender cómo los stakeholders acceden a informaciones, productos y servicios (Bashir, Papamichail \& Malik, 2017), o hacen comentarios relativos a estos. Estas herramientas abarcan el análisis de redes sociales y de los espacios de interacción que se habilitan en las compañías, los cuales son útiles para crear y expandir mecanismos de escucha y 
respuesta o comunidades virtuales para la innovación (Awazu et al., 2009; Schröder \& Hölze, 2010; Scuotto, Del Giudice, Della Peruta \& Tarba, 2017; Von Platen, 2015).

- Participando en la creación de plataformas basadas en tecnologías digitales, en las que las diferentes partes que aportan a la ICA puedan trabajar en red, compartir conocimiento, depositar ideas que circulen y conseguir que estas estén a disposición de quien las necesite (Conrado et al., 2017).

\section{Propuesta para un marco de relaciones}

En consideración a los aspectos hasta aquí contemplados, la relación que se propone que existe entre ICA y comunicación estratégica, se presenta en la figura 1. El contexto general de la relación es el entorno en el que se inscriben la organización y los grupos de interés que pueden tener interacciones periódicas, si son stakeholders primarios ${ }^{8}$ o puede tratarse de grupos que, al estar en la esfera pública, pueden participar, en cualquier momento, en las discusiones que vinculan las actuaciones de las compañías. La relación no se tiene con el entorno, como ente inmaterial, sino con personas y grupos. Estos grupos se han demarcado en el óvalo que aparece al lado izquierdo del esquema. Los dos óvalos insertados en el entorno se han trazado con líneas punteadas que simulan membranas por las que transitan ideas y nuevos desarrollos o aplicaciones.

En el entorno se asientan diversos grupos que participan en el desarrollo de la innovación en las compañías: el mercado, del que hacen parte los consumidores, otras compañías del sector de negocios, los proveedores y agentes de la cadena productiva o de generación de valor, que pueden ser los distribuidores, así como la competencia, la cual no siempre tiene que ser tomada como un contrario, sino, a veces, como aliada o cliente, puesto que puede llegar a ser potencial compradora o vendedora de innovaciones de entidades que operan negocios afines.

8 Para algunos autores (Patzer, Voegtlin \& Scherer, 2018), los stakeholders primarios son los que tienen una influencia directa en el cumplimiento de las metas de la organización. En este grupo, incluyen a empleados, accionistas, compradores y proveedores. Las organizaciones no gubernamentales, las organizaciones internacionales y los medios de comunicación son considerados stakeholders secundarios. 


\section{Figura 1. Relaciones entre ICA y comunicación estratégica.}

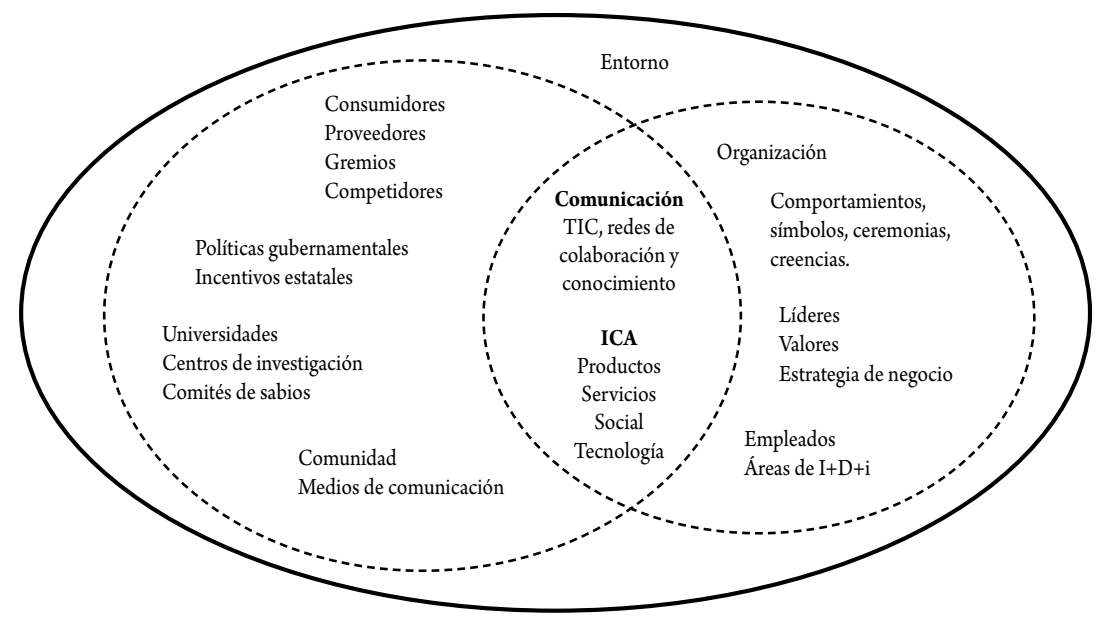

Fuente: elaboración propia.

La innovación puede ser impulsada o apoyada desde políticas públicas y las empresas pueden recibir incentivos estatales que la benefician. También en el entorno aparecen grupos de actores que han tenido un papel protagonista en la innovación debido a su vocación investigativa. Estos incluyen universidades y centros de investigación (Dalmarco, Zawislak, Hulsink \& Brambilla, 2015; Scandura, 2016; Shinkevich et al., 2016) que participan como consultores o reciben una retribución directa mediante la firma de convenios o alianzas.

Algunas organizaciones pertenecen a redes institucionales que están compuestas no solo por entidades formales, sino también por grupos informales, como activistas y colectividades de consumidores críticos. La empresa no puede gestionarse a espaldas de esos grupos, por lo que una fuerte orientación hacia las redes o la pertenencia a estas asegura que las organizaciones estén más ajustadas a la sociedad. Para representar a estos grupos, se han contemplado en la figura 1 los medios informativos que pueden actuar como legitimadores de las innovaciones de la compañía, a la par que el público en general, que aporta desde sus opiniones e ideas, sin necesidad de ser cliente o usuario, pero que puede incidir en aspectos relacionados con las afectaciones que las empresas hacen a los valores sociales o al medio ambiente. 
Antes de entrar a describir la parte central de la figura 1, es pertinente dar cuenta de los elementos que aparecen en el óvalo de la derecha, relativos al contexto interno de la organización. Allí se discriminan tres grupos de elementos que inciden de un modo más claro en la ICA. Por una parte, los propios de la comunicación, materia prima en la consolidación de una cultura institucional que hace énfasis en la innovación: comportamientos, símbolos, ceremonias o creencias, los cuales ayudan a dar sentido a la innovación como motor de cambio y adaptabilidad.

Por último, la estructura organizativa puede favorecer o no la innovación, en la medida en que, como se presenta en estudios sobre la relación que existe entre cultura e innovación (Urgal, Quintás \& Arévalo, 2011), haya un trabajo en red, de tipo colaborativo, en el que existen numerosos puntos de contacto entre la organización y el entorno, y no solo el que puedan tener el alto directivo, el área de $\mathrm{I}+\mathrm{D}+\mathrm{i}$ o el área de comunicaciones, sino otros empleados que participan en redes externas a la empresa, que se convierten en agentes multiplicadores de su pensamiento o ingresan en ella ideas disruptivas para convertirla en una entidad pionera o creadora.

En este mismo lado del esquema están, por último, las áreas de I+D+i que se ocupan de estas actividades, de manera específica, en la empresa. En la ICA sigue siendo relevante contar con áreas responsables de este proceso, porque garantiza que alguien se ocupe expresamente de planificarla, movilizar a otros y materializarla, o como parte fundamental de la estrategia de la compañía o como política de esta.

Los actores contenidos en la figura 1 aportan ideas, opiniones, conocimientos e información en los procesos de ICA, la cual aparece en la intersección de los dos óvalos. La circulación de saberes, conocimientos o propuestas es posible gracias a la comunicación entre entorno y organización, o viceversa. Esta puede hacerse cara a cara o utilizando las TIC, lo cual puede conducir a innovaciones tecnológicas, sociales o que se aplican a diferentes procesos organizativos. 


\section{Metodología}

El objetivo de la investigación aplicada ha sido verificar si se cumple la condición reflexiva de la comunicación estratégica de un grupo de empresas antioqueñas que cotiza en la Bolsa de Bolsa de Valores de Colombia (BVC) en los procesos de ICA, a partir de los elementos contemplados en el marco propuesto. Para dar cumplimiento a este objetivo, se han planteado las siguientes preguntas: ¿cuál es el concepto de innovación que utilizan las empresas de origen antioqueño que cotizan en la BVC?, ¿qué tipo de innovación se da en ellas?, ¿cuáles son los elementos del entorno, la organización y la comunicación que se presentan en los procesos de innovación? y żcómo apoyan las áreas de comunicaciones los procesos de innovación en este grupo de entidades?

Para contrastar el marco propuesto, con la práctica, se eligieron las primeras cuarenta empresas de origen nacional que aparecen en el índice bursátil de referencia Colcap $(2017)^{9}$ y que cotizan en la BVC, para invitarlas a participar en la investigación. Este criterio primó en la definición de la muestra, por cuanto la investigación abarcó empresas de diferentes ciudades del país. Otra razón que justifica la elección es que a estas compañías se les exige mayor solidez y transparencia en las informaciones que entregan al mercado de valores, así como reportar sus estados financieros para sus accionistas y otros interesados en invertir en ellas, lo que hace suponer que dan relevancia a sus actividades de comunicación.

En este artículo se presentan los resultados que arrojaron las entrevistas realizadas con ocho de ellas que tienen su casa matriz en el departamento de Antioquia, pero sus operaciones se desarrollan en diversos países de América Latina. Si bien todas se originaron en esa región de Colombia, algunas de ellas cuentan con capital extranjero (Almacenes Éxito, Grupo Familia). Las únicas compañías de esta región que accedieron a atender las entrevistas hacen parte de la muestra final que se presenta en la tabla 2. Allí

9 "El Colcap es uno de los índices bursátiles de la BVC o Bolsa de Valores de Colombia (sociedad anónima de carácter privado, en la que se inscriben y negocian valores públicamente), este indicador refleja las variaciones de los precios de las 20 acciones más líquidas donde el valor de Capitalización Bursátil ajustada de cada compañía determina su participación dentro de dicho índice. Su valor inicial fue de 1.000 puntos y el primer cálculo se realizó el 15 de Enero de 2008” (Rankia, 2019). 
mismo se incluyen los perfiles de las personas que participaron en la investigación y los ámbitos geográficos de actuación de las compañías.

\section{Tabla 1. Empresas de origen antioqueño invitadas a participar en el estudio}

\begin{tabular}{|c|c|}
\hline Empresa & Sector \\
\hline Almacenes Éxito & Consumo masivo, supermercados \\
\hline Bancolombia & Banca \\
\hline Celsia & Energía \\
\hline Cementos Argos & Infraestructura \\
\hline Compañía de Empaques & Fibras naturales y resinas plásticas \\
\hline Construcciones El Cóndor & Infraestructura y construcción \\
\hline Enka & Producción de polímeros y fibras sintéticas \\
\hline Estra & Plásticos para el hogar y la industria \\
\hline Fabricato & Textil \\
\hline Grupo Argos & Matriz de inversiones en infraestructura \\
\hline Grupo SURA & Inversiones, servicios financieros, seguros de vida y salud \\
\hline Grupo Familia & Consumo masivo, higiene \\
\hline Grupo Nutresa & Consumo masivo, alimentos \\
\hline Grupo Orbis & Pinturas, químicos, agua y comercio \\
\hline Interconexión Eléctrica S. A. E. S. P. Isa & Transporte y conexión de energía eléctrica, vías y comunicaciones \\
\hline Isagen & Generación de energía \\
\hline Mineros S. A. & Exploración y explotación minera de oro \\
\hline Protección & Fondo de ahorro en pensiones y cesantías \\
\hline Valores Simesa S. A. & Inversiones \\
\hline
\end{tabular}

Fuente: elaboración propia con datos obtenidos de Bvc.com (s. f.).

\section{Tabla 2. Muestra final}

\begin{tabular}{|l|l|l|}
\hline \multicolumn{1}{|c|}{ Empresa } & \multicolumn{1}{|c|}{ Cargo de la persona entrevistada } & \multicolumn{1}{c|}{ Ámbito geográfico de actuación } \\
\hline Almacenes Éxito & $\begin{array}{l}\text { Directora de Comunicaciones } \\
\text { Externas } \\
\text { Director de Innovación }\end{array}$ & $\begin{array}{l}\text { Colombia (Grupo Éxito), Uruguay (Disco y Devoto), } \\
\text { Brasil (Grupo Pao de Azucar) y Argentina (Libertad) }\end{array}$ \\
\hline Celsia & Director de Comunicaciones & Colombia, Panamá y Costa Rica \\
\hline Cementos Argos & Gerente de Comunicaciones & $\begin{array}{l}\text { Estados Unidos, Honduras, Panamá, Haití, Venezuela, } \\
\text { Curazao, Surinam, Guayana Francesa, República } \\
\text { Dominicana, Puerto Rico, Santo Tomás, San Martín, } \\
\text { Innovación y el Desarrollo }\end{array}$ \\
\hline
\end{tabular}




\begin{tabular}{|c|c|c|}
\hline Empresa & Cargo de la persona entrevistada & Ámbito geográfico de actuación \\
\hline Fabricato & Jefe de Desarrollo Humano & Colombia \\
\hline Grupo Argos & $\begin{array}{l}\text { Gerente Gestión de Negocios } \\
\text { Gerente de Sostenibilidad }\end{array}$ & $\begin{array}{l}\text { Colombia y países donde operan las empresas que } \\
\text { hacen parte del Grupo (Celsia, Cementos Argos y } \\
\text { Odinsa) }\end{array}$ \\
\hline Grupo Familia & $\begin{array}{l}\text { Coordinadora de Cultura Corporativa } \\
\text { Analista de Comunicaciones } \\
\text { Corporativas }\end{array}$ & $\begin{array}{l}\text { Colombia, Ecuador, Puerto Rico, Argentina, Chile, } \\
\text { Perú, Bolivia, República Dominicana }\end{array}$ \\
\hline Grupo SURA & $\begin{array}{l}\text { Gerente de Comunicaciones e } \\
\text { Identidad Corporativa }\end{array}$ & $\begin{array}{l}\text { Argentina, Brasil, Chile, Colombia, El Salvador, México, } \\
\text { Panamá, Perú, República Dominicana, Uruguay }\end{array}$ \\
\hline $\begin{array}{l}\text { Interconexión } \\
\text { Eléctrica S. A. E. } \\
\text { S.P. } \\
\text { Isa }\end{array}$ & $\begin{array}{l}\text { Especialista de Innovación } \\
\text { Especialista de Comunicaciones }\end{array}$ & $\begin{array}{l}\text { Colombia, Ecuador, Perú, Bolivia, Panamá, Brasil, } \\
\text { Argentina y Chile }\end{array}$ \\
\hline
\end{tabular}

Fuente: elaboración propia con datos de las entrevistas.

Como metodología, se eligió la entrevista en profundidad, porque permite obtener explicaciones de los responsables de los procesos que son objeto de estudio en la investigación, captar sus actitudes e interpretaciones sobre la manera como se relacionan entre las áreas y con sus respectivos ecosistemas de innovación, el apoyo que brindan al proceso y los retos que enfrentan en sus proyectos y cargos. Se entrevistó a los comunicadores responsables de apoyar las actividades de innovación y a los directores de las áreas de innovación o dependencias encargadas de tareas afines. A este grupo de profesionales se les aplicó el cuestionario, el cual estuvo organizado en cinco segmentos, con cuestiones que hacían posible vislumbrar el modo como se presentan los elementos del marco propositivo de relaciones entre ICA y comunicación estratégica. Las preguntas se agruparon así:

- Concepto de innovación, estructura de las áreas y tiempos para innovar. Qué es innovar para la organización, existencia de una política general de la compañía para fortalecerla, inclusión de la innovación en la estrategia del negocio, recursos destinados a gestionarla (áreas, personas, tiempos, laboratorios), beneficios que ha brindado la innovación y que han sido identificados, aspectos en los que más se percibe que se hacen innovaciones (procesos, servicios, productos) y periodicidad percibida con la que se introducen innovaciones. 
- Vigilancia del entorno. Existencia de sistemas formales para capturar información sobre avances o desarrollos en el sector de negocios, percepciones de los entrevistados acerca de la adaptación de la empresa a las exigencias del mercado, relevancia que se otorga a la introducción de nuevos productos en el mercado por parte de la organización.

- Colaboración interna. Participación de los empleados, de manera individual o colectiva, en procesos de innovación propios o ajenos a la empresa, inclusión de personal de diferentes áreas de la organización en los procesos de innovación, evidencia de comportamientos de innovación en la cultura corporativa, estímulos que reciben los empleados por innovar, autonomía de los equipos de trabajo para innovar, dedicación de tiempo de la jornada laboral a los procesos de innovación, proceso interno que surten las innovaciones que proponen los empleados, maneras de fomentar la innovación.

- Colaboración externa. Participación de actores externos en los procesos de innovación de la empresa, participación de la empresa en procesos de innovación de agentes externos, relaciones con diferentes grupos de interés en los procesos de innovación, estímulos o beneficios que reciben los grupos externos a la organización por participar en los procesos de innovación, dificultades y logros que se han presentado en los procesos de innovación realizados con externos.

- Comunicación. Existencia de plataformas digitales en las que se consignen nuevas ideas; medios de comunicación en los que se plasman y divulgan las experiencias de innovación relacionadas con la empresa; medios o recursos de comunicación sobre el desarrollo de nuevos proyectos, intercambio de experiencias, fomento de nuevas ideas y divulgación de buenas prácticas con agentes externos; apoyo de las áreas de comunicación a las diferentes etapas del proceso de innovación (recogida de iniciativas, desarrollo y materialización de las ideas, experimentación o pruebas piloto, producción y comercialización); innovación que se aplica en los procesos de comunicación; relato de experiencias exitosas sobre el apoyo de la comunicación a los procesos de innovación de las empresas; papel que desempeñan los profesionales de la comunicación como intermediarios entre la empresa y su entorno, a 
partir de la premisa de que ellos hacen parte del grupo de responsables de gestionar el ecosistema de públicos que aportan a la innovación $\mathrm{y}$ de que el intercambio de mensajes fluya en las redes de relaciones.

Las entrevistas se realizaron entre agosto y noviembre de 2018, en la ciudad de Medellín. En algunos casos, fue preciso complementarlas mediante teleconferencias. Entre las razones que se tuvieron para elegir el departamento de Antioquia como una región relevante para esta investigación, está el hecho de que la mayor parte de las compañías que figuran en el índice Colcap de la BVC, tomado como referencia para el estudio, procede de esta zona. ${ }^{10}$ Asimismo, porque su capital, Medellín, ha sido reconocida como la ciudad más innovadora del mundo. ${ }^{11}$ Allí se creará el primer Centro para la Cuarta Revolución Industrial del Foro Económico Mundial ${ }^{12} \mathrm{y}$ se invierte un $2.14 \%$ del PIB en actividades de innovación, ciencia y tecnología, por encima del promedio de otras ciudades de Colombia, que es del 0,7 \% (El Colombiano, 2019).

Antioquia es uno de los 33 departamentos en los que se divide Colombia. Tiene una superficie de $63612 \mathrm{~km}^{2}$, es decir, es más grande que Costa Rica, que tiene $51500 \mathrm{~km}^{2}$. Las principales actividades económicas de la región comprenden los establecimientos financieros, el comercio, la construcción y el agro (Restrepo, 2011). En 2017, la Cámara de Comercio de Medellín previó un crecimiento del $3 \%$ en la economía del departamento para 2018 y reportó que en 2017 allí había 39 empresas por cada 1000 habitantes. Además, y de acuerdo con el Departamento Administrativo Nacional de Estadística (DANE, 2017), Antioquia es la segunda economía con mayor participación en el PIB nacional, con un $15 \%$ después de Bogotá, la capital del país. ${ }^{13}$

10 Del listado de empresas tomado como referencia, alrededor del $50 \%$ son antioqueñas.

11 En 2013, Medellín ganó el concurso City of the Year, organizado por el diario estadounidense Wall Street Journal y Citi Group, como la ciudad más innovadora del mundo, por encima de ciudades como Tel Aviv y Nueva York, las otras dos finalistas, y fue escogida por encima de 200 ciudades de todos los continentes (Camargo, 2013).

12 La designación se hizo en el Foro Económico Mundial realizado en Davos, Suiza, el 20 de enero de 2019. La cuarta revolución industrial se caracteriza por la integración de tecnologías que difuminan las líneas entre la esfera física, digital y biológica, y está marcada por avances tecnológicos emergentes en campos como la robótica, inteligencia artificial, blockchain (o cadena de bloques), nanotecnología, computación cuántica, biotecnología, internet de las cosas e impresión en 3D (Dinero, 2019).

13 A la fecha, los entes que elaboran estos reportes no han divulgado los datos de 2018. 
En el proceso de investigación fue difícil comprometer a todas las empresas de la región que cotizan en la BVC. Como afirmaron algunos consultados, puede deberse a que reciben numerosas solicitudes para diligenciar encuestas y atender investigaciones de todo tipo, para las cuales no disponen de tiempo. Otra razón para no participar obedece al temor que existe en algunas organizaciones a comunicar información confidencial, relativa a la estrategia, la cual, como se evidencia en muchas de las respuestas, tiene que ver, la mayor parte de las veces, con sus actividades de innovación.

\section{Resultados y análisis}

Los resultados más notables de las entrevistas realizadas se presentan tomando como elementos de análisis y discusión los consignados en el mapa propuesto sobre la relación entre ICA y comunicación estratégica. Es decir, el entorno, la organización y la comunicación de la innovación. Para dar sentido a la presentación de los resultados, se expone, primero, una sección con tres elementos que dan contexto a las cuestiones centrales de la investigación: a) el concepto que tienen los entrevistados acerca de lo que es la innovación, b) los tipos de innovación que existen en las empresas y c) los ámbitos de la organización en los que esta se aplica con mayor frecuencia.

\section{Concepto de innovación, niveles y campos de aplicación}

Los consultados expresan una visión amplia acerca de lo que es innovar. Comprenden que existen diferentes maneras de hacerlo y les parece que todas ellas reportan algún tipo de beneficio. "No es únicamente Apple lanzando su teléfono nuevo" (Celsia, comunicación personal, 18 septiembre 2018), "Pueden ser adaptaciones a lo que ya hay o hacer el trabajo de manera diferente, de acuerdo con las necesidades del mercado" (Almacenes Éxito, comunicación personal, 18 septiembre 2018).

La aceptación por parte del mercado es también una condición necesaria en Fabricato (comunicación personal, 17 septiembre 2018), para que una innovación se considere como tal. Allí los empleados están proponiendo de manera constante ideas que mejoran los procesos, aunque no siempre estas ideas sean disruptivas. En Isa, en cambio, la innovación es todo aquello significativamente mejorado, siempre y cuando sea nuevo 
para la organización: "Así exista en otra parte, porque de lo que se trata es de que la innovación esté acompasada con la estrategia”. De ahí que innovar se defina en esta empresa como "un proceso deliberado, estructurado, sistemático y evolutivo, que no es fruto de la aleatoriedad" (Isa, comunicación personal, 17 septiembre 2018).

En Grupo Argos, holding de empresas de cemento, energía y concesiones viales y aeroportuarias, se destaca la utilidad práctica que les aporta la innovación, pues para ellos consiste en "la búsqueda de ampliación de los negocios actuales, con una mirada que pueda ser disruptiva y que permita desarrollar nuevos modelos de negocio para las empresas del grupo" (Grupo Argos, comunicación personal, 17 octubre 2018).

En vista de la amplitud de las propuestas que surgen día a día por parte de empleados o por imponerse desde el entorno, en Isa, Celsia y Cementos Argos, la innovación se clasifica en distintas categorías, para diferenciar las incrementales de las disruptivas. Así es como en Isa existe una pirámide en la que figuran proyectos de todo tipo. En la base aparecen el mayor número, por lo menos 1000, que no se caracterizan por necesitar un presupuesto adicional, sino que se hacen con conocimiento propio de las áreas, implican poco riesgo y se desarrollan rápido. En la parte superior, están los proyectos que representan la innovación radical, que "es aquella en la que se necesitan redes, en la que no se tiene el conocimiento tan fácil, se necesita presupuesto adicional y armar equipos de diferentes áreas $[. .$.$] Es un$ tipo de innovación que genera nuevos ingresos" (Isa, comunicación personal, 17 septiembre 2018). En el punto medio de la pirámide también hay proyectos. No tantos como en la base, pero un poco más que en la cima, donde no hay más de dos, por lo general. Y ese, dice el especialista de innovación entrevistado, "puede ser el comportamiento de las iniciativas en un año” (Isa, comunicación personal, 17 septiembre 2018).

La categorización es similar en Cementos Argos, donde los proyectos se trabajan por horizontes. Existe un primer horizonte con un nivel de incertidumbre bajo, alrededor de cuestiones conocidas. Son problemas que se resuelven con el conocimiento del que se dispone en la organización. 
En un segundo horizonte, están los proyectos con los que se busca expandir una tecnología o el core del negocio, como sería, por ejemplo, desarrollar un conocimiento que se tiene, que no ha acabado de ser socializado en los diferentes ámbitos del negocio y, en el último horizonte, estaría el desarrollo de tecnologías que no necesariamente existen y que se orientan a cambiar el core del negocio.

En Celsia, los horizontes van del uno al cuatro. El primero son las innovaciones que ya están en la organización, el segundo abarca las innovaciones que demuestran que pueden tener un mercado, en el tercero están los desarrollos que empiezan a tener un plan de negocios, una estructura que los acompañe y, por último, el horizonte cuatro es aquel en el que "se empieza a ver las cosas más raras e inesperadas, las cuales no dan dinero todavía, ni se espera que por ahora aporten a los resultados trimestrales" (Celsia, comunicación personal, 18 septiembre 2018). Este último vendría siendo algo similar a la cima de la pirámide, en el caso de Isa.

En las demás organizaciones consultadas, no se especificó si establecen este tipo de clasificaciones, aunque, como se ha afirmado, sí reconocen que existen diferencias entre las innovaciones incrementales y las disruptivas, y han identificado las áreas de trabajo en las que más se innova en la empresa. Así, en Almacenes Éxito (comunicación personal, 18 septiembre 2018), sobresalen las innovaciones en productos y servicios. Destacan las transformaciones constantes en los formatos de sus supermercados y en la oferta de servicios que no tienen sus competidores. Incluyen los que empezaron a ofrecer a los clientes gracias a la tecnología y que llevaron a que se diera una transformación del negocio del retail en Colombia. Un ejemplo son los catálogos digitales, en los cuales, entre muchas otras alternativas, se pueden incluir productos que no caben en espacios físicos. Suman para la innovación las aplicaciones para teléfonos móviles.

En materia de innovación social, Isa trabaja en un proyecto denominado Conexión Jaguar, que consiste en un programa de sostenibilidad corporativa con sus aliados técnicos, South Pole y Panthera, para contribuir a la conservación de la biodiversidad y a la mitigación del cambio climáti- 
co. Se realiza en conjunto con las comunidades campesinas y con él se busca recuperar y preservar bosques para reducir emisiones de gases de efecto invernadero (GEI) en zonas prioritarias para la protección y conexión de los hábitats naturales y corredores del jaguar en América Latina, que coinciden con los territorios donde Isa hace presencia (Isa, comunicación personal, 17 septiembre 2018 ):

Nos dimos cuenta de que cuando hay jaguar es porque todo está funcionando perfecto: hay agua, hay bosques, hay árboles, hay presas, frutas, vegetales, hay de todo. Es decir, el jaguar es el indicador de que el medio ambiente está bien. Entonces dijimos: Vamos a proteger el jaguar y nos inventamos el proyecto. El jaguar está desde México hasta Argentina. ¿Entonces cómo le vamos a manejar los corredores al jaguar, porque es nómada, para que pase? ¿Cómo vamos a hacer para que los ganaderos no lo maten? Con un proyecto para protegerlo. (Isa, comunicación personal, 17 septiembre 2018)

Esta innovación comprende vender bonos de carbono, que hacen posible que Isa capte ingresos que no tenía antes para proteger el jaguar. Allí mismo advierten que introducir nuevos productos no hace parte de su estrategia de innovación, porque el de ellos no es un negocio material, ya que se dedican al transporte de kilovatios. "En nuestro caso innovar es una forma nueva de hacer el trabajo, de cimentar, de lograr la consolidación financiera del grupo, de gestionar activos, de pensar en cómo serán las subestaciones del futuro y también hacemos innovaciones en servicios" (Isa, comunicación personal, 17 septiembre 2018). Lo de ellos, dice el entrevistado, es muy específico, y por eso no les parece acertado que los comparen con los mismos indicadores que se aplican en otros sectores cuando se trata de hacer listados en los que miden la innovación.

Un ejemplo de innovación en un proceso administrativo ha sido la reducción de formatos en Celsia (comunicación personal, 18 septiembre 2018), donde hasta hace poco todas las solicitudes debían hacerse diligenciando plantillas físicas. "En este momento se ha quitado el $80 \%$ de los formatos y se ha ganado en contactos directos y auténticos con las personas". De esa manera, innovar "no solo está en los productos, sino también en los procesos administrativos, en la operación normal, y ese tipo de prácti- 
cas nos ha servido para dar el mensaje a los colaboradores de Yo también lo puedo hacer" (Celsia, comunicación personal, 18 septiembre 2018), es decir, de incentivar una cultura de la innovación.

Cementos Argos ocupó el primer puesto en el ranking de innovación de la ANDI y Dinero. De acuerdo con el listado, más del $20 \%$ de los ingresos totales de esta empresa se originan en innovaciones (Dinero, 2018). En la misma publicación, destacan sus procesos de internacionalización, diversificación, su estrategia de marca y el lanzamiento constante de productos y servicios con los que atiende las necesidades de sus clientes. En 2017, sacó 115 productos nuevos al mercado, cifra que también aporta la gerente de comunicaciones en la entrevista.

En esta primera sección de resultados, las definiciones sobre innovación se ajustan a la innovación interna, que es más cerrada. Una forma cercana a la ICA se da en el proyecto Conexión Jaguar, de Isa y sus asociados. En este caso, hay entradas de conocimiento sobre el hábitat del jaguar y cómo afecta la operación de la empresa, y salidas en lo que concierne al conocimiento de Isa sobre la venta de bonos de carbono, de manera que todos los involucrados se benefician de los conocimientos compartidos.

\section{Relaciones con el entorno}

En las organizaciones que hacen parte de la muestra, existen personas encargadas de analizar el ecosistema de innovación de modo constante, para identificar ámbitos de los cuales conviene nutrirse. Ello lo hacen viajando a otros países para compararse mediante la pertenencia a redes o a partir de ejercicios de vigilancia tecnológica. En los casos en los que existe un área de innovación formalmente constituida (Almacenes Éxito, Grupo SURA, Celsia, Isa, Cementos Argos, Grupo Argos, Grupo Familia), la vigilancia tecnológica y la referenciación la hace, por lo general, el personal de estas dependencias, lo que no descarta que existan otros agentes en las demás áreas, que se ocupen del asunto, animados por mejorar su trabajo.

En las entrevistas, se consultó de manera puntual por los grupos de interés con los que se desarrollan actividades de innovación conjunta, para identificar si estos procesos merecían clasificarse como ICA. Al analizar la 
información recabada, los consultados coinciden en que la mayor parte de las relaciones que se establecen con grupos de interés externos a la organización, respecto de la colaboración abierta orientada a la innovación, son los "actores lógicos del ecosistema de innovación" (Cementos Argos, comunicación personal, 10 septiembre 2018), para referirse a proveedores, universidades, redes en las que participan, laboratorios o centros de investigación y pensamiento, y no tanto a los clientes, la comunidad en general o los competidores.

Los proveedores son el grupo con el que se hace un trabajo más cercano a la ICA, pues en la relación con ellos se destaca el aporte de ideas que nutren ambos lados de los negocios y que, además de agregar valor, son una prueba fehaciente de que la interacción impulsa la competitividad de ambas partes. Al respecto, los entrevistados consideran que es muy probable que una innovación en el proveedor impacte la manera como se hace el trabajo en la empresa que actúa como cliente, o a la inversa, que desde la empresa cliente se impacte al proveedor, porque "cualquier mejora involucra, necesariamente, que ellos participen" (Cementos Argos, comunicación personal, 10 septiembre 2018). Es el caso de las compañías de químicos para Fabricato (comunicación personal, 17 septiembre 2018), las cuales cuentan con laboratorios, y constantemente les están proponiendo ajustes que convienen a ambos y que implican hacer transformaciones en los procesos tradicionales. Fabricato reduce costos al no tener que invertir en laboratorios propios y es la relación la que le permite contar con este beneficio. Así se trate de una relación comercial, si es buena, aporta a la mejora en los procesos de la empresa cliente.

En Cementos Argos, Grupo Argos y Grupo SURA, se manejan esquemas de alianzas o joint ventures con inversionistas, siempre que los nuevos negocios revistan utilidad para ambas partes. Este es otro ejemplo de ICA, pues, "cuando se habla de compensación en este tipo de esquemas, mientras se desarrollan, se determinan reglas y roles claros de parte y parte, $y$ en la medida en que haya utilidades y durante el tiempo que exista la sociedad, habrá participación" (Cementos Argos, comunicación personal, 10 septiembre 2018). Normalmente, estas iniciativas demoran unos años 
para consolidarse, por lo que, según el caso, se evalúa si se sigue participando o si es mejor retirarse, cuando les compren la empresa o ellos quedan con el carácter de clientes o usuarios.

Las universidades nacionales y extranjeras son un grupo significativo para el trabajo de innovación de este grupo de empresas, porque, en general, son consideradas fuentes de nuevas ideas. Isa (comunicación personal, 17 septiembre 2018) cuenta con la asesoría del Massachusetts Institute of Technology (MIT) para recibir información constante sobre desarrollos que impacten en sus negocios y el Grupo Familia (comunicación personal, 16 agosto 2018) para fortalecer los indicadores de impacto social de la Fundación Familia. Isa, SURA, Éxito, Familia y Cementos Argos han participado en iniciativas de coinnovación abierta con universidades, utilizando concursos o recursos como Interacpedia (2019), una plataforma digital creada en Antioquia y con proyección global, con la que se busca que grupos de estudiantes universitarios resuelvan retos que les proponen estas compañías.

Dentro de los actores tradicionales del ecosistema de innovación, están los organismos de ciencia y tecnología. El más reconocido, porque es el que más se menciona (Cementos Argos, Fabricato, Celsia, Grupo Familia, Isa), es Colciencias. Se le cita por los incentivos que aporta en este tipo de procesos, consistentes en exención de impuestos, ayudas por concepto de regalías en las regiones o por las partidas que entrega en convocatorias en las que participan múltiples actores del país y que tienen como reconocimiento dinero, becas de formación del talento o impulso a proyectos con capital intelectual. Más que tratarse de una mutua colaboración entre la entidad y la empresa, Colciencias se ocupa de hacer intermediación entre diferentes sectores que se ven beneficiados. En Fabricato, les apoyó en la creación de Ideacción, la plataforma para que los empleados plasmen sus ideas, la cual se utiliza en la actualidad con los mismos fines, y Tecnova, organización creada por universidades y empresas del país, que formó a un grupo de empleados como líderes de innovación. ${ }^{14}$ Otra de las entidades que se menciona (Isa, comunicación personal, 17 septiembre 2018), si bien con

14 Tecnova es una organización con sede en Medellín que se orienta a apoyar organizaciones de diversos sectores en la ejecución de políticas públicas, conectando la demanda con la oferta de productos en ciencia, tecnología e innovación (CTI). 
una frecuencia mucho menor, es Innpulsa, entidad del Gobierno Nacional creada para apoyar y promover el crecimiento empresarial jalonado por la innovación. En las entrevistas, no se habló de otras entidades que hicieran parte de la estructura que el Estado colombiano pone a disposición de entidades de diferente tipo para promover este tipo de actividades, ni de otras privadas de orden nacional.

Isa y Cementos Argos participan en redes nacionales e internacionales de colaboración para recibir transferencia de conocimiento, intercambio y mucha referenciación sobre iniciativas en las que vienen trabajando pares o colegas en otras partes del mundo, orientadas a la mejora de prácticas en las industrias. En el caso del Grupo Argos, se han contratado consultores externos en el pasado, para asesorarse acerca de cómo desarrollar alianzas e invertir en spin-offs, pero en este momento han decidido hacerlo con talento del propio holding. En Celsia y SURA, se cuenta con este tipo de expertos para contar con un acompañamiento externo (tabla 3 ).

\section{Tabla 3. Grupos de interés con los que se intercambia conocimiento para generar innovaciones}

\begin{tabular}{|c|c|c|c|c|c|c|c|c|c|c|}
\hline Empresa & 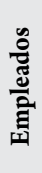 & & 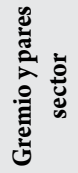 & 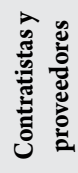 & 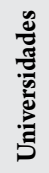 & 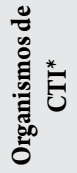 & 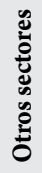 & 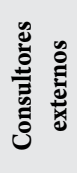 & 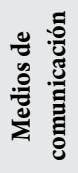 & 胥 \\
\hline Almacenes Éxito & $\mathrm{x}$ & & $\mathrm{x}$ & $\mathrm{x}$ & $\mathrm{x}$ & $\mathrm{x}$ & $\mathrm{x}$ & $\mathrm{x}$ & $\mathrm{x}$ & \\
\hline Celsia & $\mathrm{x}$ & & & $\mathrm{x}$ & $\mathrm{x}$ & $\mathrm{x}$ & & $\mathrm{x}$ & $\mathrm{x}$ & $\mathrm{x}$ \\
\hline Fabricato & $x$ & $\mathrm{x}$ & $x$ & $x$ & & $x$ & $\mathrm{x}$ & & & \\
\hline Isa & $\mathrm{x}$ & $x$ & $\mathrm{x}$ & $\mathrm{x}$ & $\mathrm{x}$ & $x$ & & $\mathrm{x}$ & $\mathrm{x}$ & $\mathrm{x}$ \\
\hline Grupo Argos & $\mathrm{x}$ & & & $\mathrm{x}$ & & & $\mathrm{x}$ & & & \\
\hline Grupo SURA & $\mathrm{x}$ & $\mathrm{x}$ & $\mathrm{x}$ & & $\mathrm{x}$ & $\mathrm{x}$ & $\mathrm{x}$ & & $\mathrm{x}$ & \\
\hline Grupo Familia & $\mathrm{x}$ & & & & $\mathrm{x}$ & $x$ & & $\mathrm{x}$ & & \\
\hline Cementos Argos & $\mathrm{x}$ & & & $x$ & $x$ & $\mathrm{x}$ & & & & \\
\hline
\end{tabular}

*Organismos de CTI: ciencia, tecnología e innovación. Pueden ser públicos o privados.

Fuente: elaboración propia a partir de información obtenida en las entrevistas. 
Con los clientes y la comunidad en general ha habido, más bien, ejercicios de una sola vía, para comunicarles resultados (Cementos Argos, comunicación personal, 10 septiembre 2018; Isa, comunicación personal, 17 septiembre 2018), algo que en Colombia se ha llamado "rendición de cuentas" y que se dirige a todos los grupos de interés, a la manera del Global Reporting Initiative (GRI). Esta es una actividad que, en ocasiones, se plantea como una conversación cara a cara. Pese a ello, sobre innovación, no se mencionan ni destacan relaciones de trabajo colaborativo que sean constantes y sistemáticas para involucrar a estos dos públicos en el desarrollo de innovaciones. Si bien algunos entrevistados afirman que les hacen consultas a los clientes, directamente desde las áreas comerciales (Almacenes Éxito, comunicación personal, 18 de septiembre 2018; Fabricato, comunicación personal, 17 de septiembre), en Cementos Argos (comunicación personal, 10 septiembre 2018) se advierte que "puede haber ejemplos de desarrollos colaborativos con los grupos impactados para tratar de encontrar soluciones", pero no se sabe con certeza qué tan metódico han sido en cuanto al modelo de desarrollo de la ICA en particular.

En la tabla 3, se presenta una relación entre las empresas y los grupos de interés con los que las organizaciones consultadas mantienen un intercambio mayor de conocimiento en asuntos que atañen a la innovación.

Para las áreas de comunicación, los proveedores son el grupo de interés que más se menciona en actividades de innovación. Esta surge espontáneamente y no como producto de una búsqueda o acuerdo. Se citan casos particulares, como la realización de eventos, los servicios que se reciben de las agencias de comunicación y el trabajo con los medios periodísticos, el cual se ha empezado a diversificar por la presencia de plataformas tecnológicas para llegar a los públicos de maneras más ajustadas a las nuevas modalidades de uso y consulta de medios. Esto demanda, muchas veces, revisar la forma como se hacen llegar contenidos a los editores y periodistas, para que los puedan aprovechar mejor (Almacenes Éxito, comunicación personal, 18 de septiembre 2018; Celsia, comunicación personal, 18 de septiembre 2018; Isa, comunicación personal, 17 septiembre 2018). 
En estos casos, se dan más las entradas de conocimiento, por parte de los proveedores hacia la organización, que las salidas. Es probable que estos mismos proveedores estén aportando las mismas ideas a otros clientes, por lo que podría presumirse, ayudan a hacer más competitiva la tarea de los comunicadores, si bien lo podrían llegar a hacer con todos sus clientes por igual. Los consultados no especifican si hacen aportes de nuevo conocimiento a sus interlocutores en este tipo de procesos.

\section{Organización}

\section{La innovación en la estrategia del negocio}

Todos los entrevistados consideran que la innovación es parte de la estrategia del negocio. En Cementos Argos, SURA y Familia, los entrevistados no se centraron tanto en si llaman innovación a un invento o a una mejora, si esta es incremental o disruptiva, sino en la incidencia que esta tiene en la estrategia de la empresa. En consecuencia, es posible afirmar que, al dar tal relevancia a este elemento, el campo estaría abonado para que las empresas consultadas desarrollen una cultura de la innovación.

De ese modo, para Cementos Argos (comunicación personal, 10 septiembre 2018), la innovación "es una capacidad institucional [ ... t transversal, que debemos consolidar y fortalecer porque apalanca la consecución de objetivos hacia el cumplimiento de la estrategia”. En el Grupo Familia (comunicación personal, 16 agosto 2018), es la plataforma que les permite alcanzar un crecimiento acelerado para generar bienestar y salud en sus consumidores y, en SURA (comunicación personal, 19 noviembre 2018), es "uno de los pilares estratégicos [... ] en este momento. Ha venido adquiriendo una importancia cada vez mayor porque en el grupo se tiene mucha conciencia sobre la necesidad de transformar los negocios a la luz de las nuevas realidades del entorno, dados los riesgos y las oportunidades que presenta la industria en la que estamos”.

En Almacenes Éxito (comunicación personal, 18 de septiembre 2018), la innovación está declarada como un valor corporativo, incluido en la planeación estratégica. En el caso de Celsia (comunicación personal, 18 sep- 
tiembre 2018), el aporte de la innovación a la estrategia apunta más que nada a diferenciarse, pues se opera en un sector en el que los competidores son vistos como agentes muy formales y, por eso, la innovación "sirve a la posición estratégica de Celsia, que es de divergencia. Admiramos y respetamos a las empresas del sector, pero no queremos ser ellas cuando seamos grandes, queremos ser otra cosa distinta. Es ahí donde la innovación forma parte del compendio estratégico para lograr eso, porque no se puede ser diferente haciendo lo mismo". Y se añade que también es un asunto que impacta el trabajo del área de comunicaciones, porque es un elemento que aporta atributos a la marca, a la manera como se espera que esta sea percibida.

\section{Estructura de la innovación}

Como se ha mencionado, en siete de las ocho entidades consultadas, existen áreas especializadas en procesos de innovación. Este hecho es un indicador de que se asignan recursos particulares a este tipo de tareas, con personas que tienen esta labor como función principal y que se ocupan de facilitar los medios para que viva y tenga dinamismo.

En Cementos Argos (comunicación personal, 10 septiembre 2018), es una vicepresidencia. Existe hace diez años y de ella hacen parte alrededor de 30 personas. Se ocupa de instalar las prácticas que pueden ayudar a gestionar ideas innovadoras en la organización y desarrollar sistemas y herramientas que puedan facilitar la identificación, el acompañamiento, las pruebas y los desarrollos asociados a iniciativas para la evaluación de los resultados y las decisiones sobre el momento en el que las innovaciones se incorporan al negocio: "Son los que hablan del tema, los que lo ponen en la agenda de conversación, los que están todo el tiempo viendo cuáles son los mejores mecanismos para desplegar lo que se debe probar y el cumplimiento de los pasos necesarios para ver si, efectivamente, vale la pena continuar trabajando en las nuevas ideas" (Cementos Argos, comunicación personal, 10 septiembre 2018).

La relación entre la dependencia especializada en innovación y las demás áreas es bastante similar en todos los casos, pues, como en Cementos Argos, en Celsia el área de innovación se encarga de estructurar los horizontes y explorar nuevas líneas de trabajo. Además, en cada área de la or- 
ganización, existe interés en materializar una actitud innovadora entre los empleados. Desde el comité directivo se plantean situaciones a las que se debe encontrar soluciones. El área de innovación lidera el trabajo con las demás dependencias en la resolución de los problemas y, para ello, en algunos casos, conforman células con personas de diferentes especialidades, que son empleados de la empresa, de tal forma que un problema pueda ser valorado desde distintas miradas. Algunas personas se retiran de sus actividades cotidianas durante un semestre, un día a la semana, o el tiempo que se requiera, para participar en estos grupos.

En el Grupo Familia (comunicación personal, 16 agosto 2018), existe una unidad de $\mathrm{I}+\mathrm{D}+\mathrm{i}$, la cual participa en los procesos de transformación de los negocios. Allí, la inversión en innovación "no solo se traduce en recursos económicos, sino también en intangibles, como son tiempo, creación de competencias y conocimiento".

En Almacenes Éxito (comunicación personal, 18 septiembre 2018), la dirección de innovación depende de la vicepresidencia de operaciones. Su misión es "insertar la compañía dentro del ecosistema de innovación del país, de las start-ups, y empezar a generar alianzas”, y aunque es un área más bien nueva, es claro que en ella se encargan de dar estructura a los proyectos que luego deberán desarrollarse en las áreas. Quien la dirige "debe generar una dinámica de integración $[\ldots]$, porque, entre otras razones, no hay un presupuesto que diga Innovación y desarrollo. Ese es un presupuesto que tiene cada una de las áreas, las cuales lo destinan a tecnología, software, nuevos desarrollos" (Almacenes Éxito, comunicación personal, 18 septiembre 2018).

El área de innovación hace parte de la estructura organizacional de Isa desde 2014. Ello no ha impedido que esta sea una empresa innovadora desde su fundación:

Sí ha habido grandes hitos innovadores, pese a no haberla tenido dentro de la estructura, como fue vender Acciones para Todos. Ese fue un asunto muy novedoso. Después lo hicieron en Ecopetrol, pero fuimos los primeros. También fuimos los primeros que, siendo una 
empresa del Estado, salimos a comprar empresas a otros países. Salimos a comprar empresas a Brasil [...] y hoy en día tenemos más kilómetros de línea en Brasil que en Colombia. (Isa, comunicación personal, 17 septiembre 2018)

En el Grupo SURA (comunicación personal, 19 noviembre 2018), la dirección de innovación hace parte de la gerencia de planeación. Se ocupa de gestionar los procesos de innovación propios del holding, traza lineamientos que son útiles para compartir conocimientos e iniciativas y para crear sinergias entre los negocios, pues "la dinámica propia de innovación de los negocios sucede. No es que haya una sola área de innovación, porque hay innovación en cada país, sino que lo que hace se vuelve operativo de manera transversal, como un proceso que es integral en las compañías y que está muy pegado a los negocios, porque es en ellos donde se identifica la necesidad de innovar, ya sea en canales, productos, servicios, o con el apoyo de la tecnología”.

En Grupo Argos (comunicación personal, 17 octubre 2018), el área que se ocupa de la innovación es la Gerencia de Gestión de Negocios que se concentra, en este momento, en el desarrollo de un programa de joint ventures y trabajo con aliados. En Fabricato (comunicación personal, 17 septiembre 2018), no existe una dependencia formalmente constituida para concentrar la gestión de la innovación o para movilizarlos a todos en torno al tema, sino que se cuenta con el trabajo complementario de tres áreas que se ocupan, desde sus labores, de alguna actividad relativa a innovar: la dirección de operaciones (en los procesos), el área comercial (con proveedores y clientes) y desarrollo humano (con empleados y en la comunicación).

En todas las organizaciones, se hace innovación con los empleados, ya sea con células de trabajo, ya sea mediante convocatorias y concursos de ideas. En cualquier caso, en Cementos Argos (comunicación personal, 10 septiembre 2018), se menciona un grupo que para ellos es muy importante. Se trata de doce personas que tienen el título académico de doctores, reclutados de manera particular para impulsar y desarrollar iniciativas, así como para dar importancia al conocimiento”. 


\section{Cultura de la innovación}

En esta parte de la investigación, se ha buscado establecer relaciones entre la participación que tienen los empleados en la innovación y la manera como se busca fortalecer una cultura innovadora entre ellos. Al respecto, se ha consultado por rituales, ceremonias, creencias y comportamientos cotidianos que reflejen la existencia de una cultura de la innovación. Así, el Grupo Argos (comunicación personal, 10 septiembre 2018) pone el foco, por ahora, en sacar proyectos, para que a partir de ellos se fortalezca la cultura, lo que se interpreta como "a innovar se aprende innovando".

Un primer problema identificado en Fabricato (comunicación personal, 17 septiembre 2018), relativo a crear una cultura de innovación, consiste en que esta sea permanente, en el sentido de que la gente esté pensando, siempre, en cómo plantear nuevas ideas o mejorar procesos. La persona entrevistada opina que esta es una cuestión muy difícil de sembrar $y$, sobre todo, de mantener, porque los seres humanos, en general, siempre buscan una recompensa inmediata, quieren transformarlo todo $\mathrm{y}$, al final, se desilusionan porque los logros no son tan radicales como se pensaba, o porque no hay paciencia para el proceso, que muchas veces es de largo plazo. Otro problema es mantener vivo el tema en las conversaciones, en los medios internos, para que la innovación sea vista como un esfuerzo constante de parte de los empleados, porque "no es un asunto que se mueva solo, hay que estarlo moviendo [... ] Y cuando uno habla sobre él le dicen: ¿Revivieron el programa? Pero es que el programa no se había muerto, solo lo estamos recordando" (Fabricato, comunicación personal, 17 septiembre 2018 ).

El Grupo Familia (comunicación personal, 16 agosto 2018), cuenta con una política institucional orientada a favorecer la innovación. Respecto de su inserción como valor en la cultura, existe un principio que hace parte de su declaración ideológica, que es “Innovamos para ganar”. En relación con este lema, las personas entrevistadas destacan tres tipos de comportamientos que se esperan de los miembros de la organización: a) anticiparse a nuevas oportunidades y desarrollarlas con valentía y disciplina, asumiendo riesgos y capitalizando los errores; b) actuar con apertura al cambio, ex- 
plorando nuevas formas de hacer las cosas y adaptándose con facilidad; y c) identificar nuevas tecnologías evolucionando el mundo digital.

Para Isa (comunicación personal, 17 septiembre 2018), la innovación es un rasgo distintivo del grupo y lo ponen a prueba constantemente: "a pesar de la aversión al riesgo, porque el miedo a equivocarse compite con la cultura de innovación, en la que también hay que aprender a fallar y a recuperarse rápido". En la misma dirección trabajan en el Grupo SURA (comunicación personal, 19 noviembre 2018), donde consideran que han avanzado mucho en los últimos años, pues de manera consciente han venido promoviendo múltiples dinámicas que les ayudan a fortalecer la cultura de innovación: "Esto es un proceso. Pero creo que se ha avanzado sustancialmente $[\ldots]$ Permanentemente nos están motivando a salirnos de la caja, en las formas de pensamiento".

En Almacenes Éxito (comunicación personal, 18 noviembre 2018), la innovación es vista como una necesidad, en particular por la alta exposición de sus servicios, porque la base de la compañía son los supermercados. Respecto de los comportamientos de la organización que llevarían a afirmar que tiene una cultura de innovación, la comunicadora entrevistada señala que "hoy el tema está en la alta dirección, todo el mundo está hablando de innovación [...] Yo voy a hacer la asamblea de accionistas y mi jefe me dice: ¿Qué hay de nuevo? ¿Cómo la vamos a hacer de una manera diferente?". Ese, dice, es un reto permanente de la comunicación: “¿Cómo contar que se inaugura un supermercado, para que sea noticia en los medios, cuando no es la primera vez que se hace y cuando se envían noticias a los mismos periodistas?".

En Celsia, existe la certeza de que esta no es una tarea exclusiva del área que tiene el nombre, pues se premian y reconocen las ideas que aportan cambios, en asuntos muy simples. Su esquema es similar al de Cementos Argos, ya que, así como en Celsia tienen un núcleo de experimentación en temas de almacenamiento de energía y energía solar, en Yumbo, en el departamento del Valle del Cauca, en Cementos Argos tienen el Laboratorio Argos para la Innovación y el Desarrollo, una estructura de seis pisos, ubicada en la Universidad Eafit, en la ciudad de Medellín, que tiene como 
propósito "ser punto de encuentro de diferentes personas que trabajan en la innovación" (Cementos Argos, comunicación personal, 10 septiembre 2018), y que no está dirigida solo a los empleados de esa empresa o a personas interesadas en desarrollar innovaciones a partir del cemento, sino a todo aquel que esté interesado en innovar, sin importar el ámbito.

Se ha encontrado que en las organizaciones de la muestra priman los eventos para dar visibilidad a estas temáticas y las ceremonias de reconocimiento a las iniciativas de los empleados. De este modo, en Isa tienen las Jornadas del Conocimiento, cada dos años. En ellas participan empleados de todas las filiales, que viajan a Medellín a presentar innovaciones que hayan implementado en sus trabajos, muestran cómo van y de qué modo han mejorado. La última vez participaron 1055 empleados, en ocho categorías. "Para nosotros ese es un indicador de que tenemos una cultura de innovación” (Isa, comunicación personal, 17 septiembre 2018).

\section{Comunicación}

Como se expuso en el apartado anterior, una de las dificultades que enfrentan estas organizaciones, cuando se trata de fortalecer la cultura de la innovación, es cómo mantener vivo el tema, desde la visibilidad que se le da en los medios de comunicación institucional o hacia fuera, como parte de las noticias que las entidades generan para los periodistas, pues, por lo general, las personas orientan su percepción solo hacia una temática a la vez, y la innovación "compite" con asuntos tan importantes como la responsabilidad empresarial, la calidad, la gestión del conocimiento y las noticias del día a día, lo que configura un reto grande para la comunicación.

En el caso de la innovación, como sucede con otras temáticas, las áreas de comunicación de las empresas de la muestra se dedican a acompañar. A veces sirven de puente entre los públicos y las entidades, pero ninguna de estas dependencias tiene un rol de responsabilidad directo, tan activo como para hacer parte del grupo de trabajo permanente del área de innovación. El apoyo se brinda "por demanda", pues, como hacen notar en Isa, 
en un todo y engranarlo. Entonces, ¿vamos a hablar de innovación? No. Vamos a hablar de transformación digital apalancada por la innovación. Vamos a hablar de colaboradores apalancados en nuevos puestos de trabajo generados por la innovación. Nuevas transformaciones, derivadas de la innovación. La innovación puede ser una palanca. De pronto la matamos si comunicamos la innovación por la innovación, y si así fuera, no tendría profundidad. (comunicación personal, 17 septiembre 2018)

Para ellos, es mejor comunicar logros, asuntos finalizados, que sembrar expectativas sobre innovaciones cuyos resultados, al final, no sean los esperados. Comunicarlos antes configura una promesa. En algunos casos, la comunicación es vista como el proceso para mostrar lo que se hace: "Uno nada se gana haciendo unos programas sin tener nada concreto que mostrar, porque es muy difícil cambiar la cultura, solo diciendo que va a cambiar la cultura” (Grupo Argos, comunicación personal, 17 octubre 2018). También en el Grupo Familia (comunicación personal, 16 agosto 2018) la tarea de comunicaciones consiste en facilitar, asesorar y acompañar el proceso de innovación, mediante el uso de medios de comunicación. Además, cada año realizan dos o tres campañas en las que participan los empleados, y que responden a los retos de la sostenibilidad y el mercado en materia de innovación.

En Celsia, las preocupaciones se asocian a los retos que les impone la innovación cuando se hace con terceros, y los socios externos la comunican sin que se haya todavía un resultado, se haya trazado un acuerdo previo acerca de cómo figurarán las marcas o qué parte de los hallazgos serán divulgados. En esta empresa, comentan que estos procesos se mueven tan rápido que, en ocasiones, los profesionales de la comunicación no alcanzan a participar cuando se convierten en noticia, por lo que, a veces, quienes intervienen desde las áreas técnicas, pasan por alto que también allí se requiere una adecuada gestión de la marca. El interés de los profesionales de la comunicación, dicen, no es entorpecer la innovación, sino capitalizarla para la buena reputación de la empresa. En este caso, se requiere pensar la gestión de la marca en un mundo que funciona en red, en programas y productos que son resultado de trabajos conjuntos, algo acerca de lo cual se maneja, en algunas entidades (Isa, Cementos Argos), con políticas de pro- 
piedad intelectual, que son muy formales y que no alcanzan a dictar normas concretas sobre visibilidad y presencia de la marca.

Los públicos prioritarios con los que se relacionan los comunicadores consultados son los empleados, para la comunicación interna, y los periodistas especializados de cada sector, en lo que respecta a los stakeholders externos. En el caso de la relación con la comunidad, las áreas que proponen y llevan las conversaciones son las de sostenibilidad. Asimismo, los comunicadores manejan los sistemas de medios en sus respectivas entidades, y no tienen una injerencia directa en el diseño o el mantenimiento de la información en las plataformas digitales en las que los empleados consignan ideas o nuevas propuestas para innovar. Ideacción, que es el nombre de la plataforma que se utiliza en Cementos Argos y Fabricato para recoger iniciativas de los empleados, tiene personal que se dedica a gestionarla. Estas plataformas no se consideran medios informativos por parte de los profesionales de la comunicación, sino recursos con los que se cuenta para apoyar la recogida de ideas, si bien sirven para democratizar y compartir el conocimiento.

El papel de la comunicación en cada una de las etapas del proceso de innovación (recogida de ideas, materialización de iniciativas, pruebas piloto, producción y comercialización) es, mayoritariamente, divulgar y apalancar otros sistemas de información que existen (Cementos Argos, comunicación personal, 10 septiembre 2018). Las campañas se ejecutan para hacer ver que la innovación puede ser tarea de cualquier persona, destacar logros e insistir con este tipo de mensajes, en que no solo los doctores innovan. En la tabla 4 , se presenta una síntesis de las actividades y medios más utilizados para entablar conversaciones con los grupos de interés en torno a la innovación.

En el Grupo SURA, han trabajado más por tener una presencia activa desde el punto de vista de la marca en escenarios que son relevantes para los temas asociados a la innovación, como eventos externos que organizan entidades como Ruta N. La red empresarial colaborativa que usan es Yammer y en ella los empleados comparten sus iniciativas. Observatorio es el espacio en el que se comparten proyectos o nuevas ideas propuestas por las áreas y que ellos consideran que son tan relevantes como para socializarlas. 
Tabla 4. Actividades y medios para intercambiar mensajes relativos a la innovación

\begin{tabular}{|c|c|c|c|c|c|c|c|c|c|}
\hline Empresa & Actividades con medios & Sitio web & Redes sociales & Intranet & $\begin{array}{l}\text { Carteleras digitales o } \\
\text { tradicionales }\end{array}$ & $\begin{array}{l}\text { Boletines internos } \\
\text { electrónicos }\end{array}$ & $\begin{array}{l}\text { Plataforma digital } \\
\text { para proponer } \\
\text { ideas innovadoras }\end{array}$ & Campañas & Otros \\
\hline $\begin{array}{l}\text { Almacenes } \\
\text { Éxito }\end{array}$ & Visitas a los medios & $\begin{array}{l}\text { Noticias sobre nuevos } \\
\text { formatos y servicios }\end{array}$ & $\begin{array}{l}\text { Twitter } \\
\text { Facebook }\end{array}$ & & $\begin{array}{l}\text { Carteleras en los } \\
\text { almacenes para } \\
\text { empleados y clientes }\end{array}$ & $\begin{array}{l}\text { Correos electrónicos } \\
\text { con noticias } \\
\text { relacionadas con } \\
\text { innovación } \\
\end{array}$ & $\begin{array}{l}\text { Concurso } \\
\text { Ideas de Éxito }\end{array}$ & $\begin{array}{l}\text { Premiación de ideas innovadoras con } \\
\text { reconocimiento a empleados }\end{array}$ & $\begin{array}{c}\text { App Éxito } \\
\text { App Carulla } \\
\text { Revista interna } \\
\text { Organización de eventos y ferias } \\
\end{array}$ \\
\hline Celsia & $\begin{array}{l}\text { Relaciones con medios } \\
\text { Ruedas de prensa } \\
\text { conjuntas con aliados }\end{array}$ & $\begin{array}{l}\text { Información permanente } \\
\text { sobre nuevos proyectos y } \\
\text { desarrollos alrededor de las } \\
\text { diferentes formas de la energía }\end{array}$ & $\begin{array}{l}\text { Conversaciones en } \\
\text { Twitter } \\
\text { Facebook } \\
\text { Instagram }\end{array}$ & $\begin{array}{l}\text { Miércoles de } \\
\text { recarga } \\
\text { Todo porque } \\
\text { hoy es viernes }\end{array}$ & $\begin{array}{l}\text { Contenidos sobre } \\
\text { innovación en la } \\
\text { empresa y otros } \\
\text { referentes }\end{array}$ & $\begin{array}{l}\text { Boletín electrónico } \\
\text { semanal incluye en } \\
\text { sus temáticas las } \\
\text { relacionadas con } \\
\text { innovación }\end{array}$ & & $\begin{array}{l}\text { Sensibilización frente a la innovación } \\
\text { Reverdece: ideas para acelerar número de } \\
\text { árboles sembrados } \\
\text { Búsqueda de agendas en la opinión pública } \\
\text { para ubicar temas de interés } \\
\text { Estands }\end{array}$ & $\begin{array}{c}\text { Cédulas de trabajo colaborativo } \\
\text { Coworking con empleados } \\
\text { Gestión de la marca, } \\
\text { co-branding } \\
\text { Reuniones de identificación de brechas con } \\
\text { grupos e interés } \\
\text { Organización de eventos }\end{array}$ \\
\hline $\begin{array}{l}\text { Cementos } \\
\text { Argos }\end{array}$ & Manejo de prensa & $\begin{array}{l}\text { Videos centrados en } \\
\text { innovaciones }\end{array}$ & $\begin{array}{l}\text { Canal de YouTube } \\
\text { Comunidad cerrada } \\
\text { en Facebook }\end{array}$ & $\begin{array}{l}\text { Digital World } \\
\text { Place }\end{array}$ & Información interna & & Ideacción & $\begin{array}{l}\text { Planes de divulgación sobre innovación: } \\
\text { convocatorias, innovadores, hallazgos }\end{array}$ & $\begin{array}{l}\text { Yammer (interno) } \\
\text { Diálogos con grupos de interés }\end{array}$ \\
\hline Fabricato & & $\begin{array}{l}\text { Innovaciones en telas, en } \\
\text { materia de estampación }\end{array}$ & Facebook & & $\begin{array}{l}\text { Resultados de } \\
\text { convocatorias } \\
\text { a concursos de } \\
\text { innovación }\end{array}$ & Boletín virtual mensual & Ideacción & & $\begin{array}{c}\text { Campañas periódicas para el aporte de } \\
\text { ideas innovadoras } \\
\text { Medios impresos } \\
\text { Células de innovación } \\
\text { Eventos de premiación y reconocimiento a } \\
\text { empleados por sus ideas } \\
\end{array}$ \\
\hline Grupo SURA & & $\begin{array}{l}\text { Declaraciones sobre } \\
\text { innovación y emprendimiento } \\
\text { en la sección "Sobre Grupo } \\
\text { SURA" }\end{array}$ & $\begin{array}{l}\text { Twitter } \\
\text { Facebook }\end{array}$ & & & $\begin{array}{l}\text { Presentación } \\
\text { permanente de } \\
\text { iniciativas de los } \\
\text { empleados }\end{array}$ & $\begin{array}{c}\text { Observatorio } \\
\text { para la gestión de } \\
\text { tendencias y riesgos }\end{array}$ & & $\begin{array}{c}\text { Células de trabajo colaborativo } \\
\text { Equipo E3 } \\
\text { Jornadas de innovación } \\
\text { Gestión de la marca } \\
\text { Participación en eventos de innovación } \\
\text { Yammer }\end{array}$ \\
\hline $\begin{array}{l}\text { Grupo } \\
\text { Familia }\end{array}$ & & $\begin{array}{c}\text { Información sobre } \\
\text { reconocimientos a la empresa } \\
\text { por su aporte a la innovación } \\
\text { en el país y perspectiva de } \\
\text { la empresa en materia de } \\
\text { innovación } \\
\end{array}$ & Facebook & $\begin{array}{l}\text { Nuevos } \\
\text { proyectos de } \\
\text { innovación }\end{array}$ & $\begin{array}{l}\text { Nuevos proyectos de } \\
\text { innovación }\end{array}$ & & Ingennio & $\begin{array}{l}\text { Desafíos estratégicos basados en } \\
\text { innovación, sostenibilidad y mercado }\end{array}$ & $\begin{array}{l}\text { Proyecto colaborativo con el MIT dirigido } \\
\text { a recicladores } \\
\text { Convocatorias para resolución de retos con } \\
\text { diferentes grupos de la sociedad } \\
\text { Revista }\end{array}$ \\
\hline $\begin{array}{l}\text { Holding } \\
\text { Grupo Argos }\end{array}$ & Envío de noticias & $\begin{array}{c}\text { Relevancia de la innovación y } \\
\text { gestión de esta en el grupo } \\
\text { Logros y retos en materia de } \\
\text { innovación } \\
\end{array}$ & $\begin{array}{l}\text { Facebook } \\
\text { Twitter }\end{array}$ & & & & & & $\begin{array}{l}\text { Relaciones interpersonales directas con } \\
\text { inversores y accionistas }\end{array}$ \\
\hline Isa & $\begin{array}{l}\text { Registros para la prensa } \\
\text { Comunicados } \\
\text { Manejo personalizado } \\
\text { de las relaciones con los } \\
\text { periodistas } \\
\text { Rondas con editores }\end{array}$ & $\begin{array}{c}\text { Difusión de proyectos } \\
\text { innovadores como Conexión } \\
\text { Jaguar }\end{array}$ & $\begin{array}{c}\text { Marca y } \\
\text { posicionamiento en } \\
\text { las redes sociales }\end{array}$ & & & & & & $\begin{array}{l}\text { Jornadas del Conocimiento } \\
\text { Reuniones con el clúster de Energía y otros } \\
\text { gremios del sector } \\
\text { Diálogos con grupos de interés } \\
\text { Participación en redes colaborativas de } \\
\text { innovación con entidades de otros páses }\end{array}$ \\
\hline
\end{tabular}

Fuente: elaboración propia con datos de las entrevistas. 
En materia de eventos, los comunicadores apoyan la realización de concursos, los cuales tienen como fin reducir costos, apostar por la generación de ideas y entregar incentivos en torno a propuestas innovadoras (Almacenes Éxito, comunicación personal, 18 septiembre 2018).

\section{Conclusiones}

Desde la primera década del siglo XXI, se han venido intensificando los estudios sobre ICA, en gran medida por la facilidad que brindan las TIC o la sociedad del conocimiento, además de otros aspectos de índole económica. En los casos estudiados, no se ha encontrado una relación fuerte entre la estructura de comunicación de las entidades, entendida como las dependencias integradas por profesionales del área, y los medios tecnológicos de los que disponen las compañías, con el proceso de innovar a partir de las relaciones que se sostienen con los grupos de interés.

El análisis de estudios previos sobre la ICA muestra una literatura proclive a la búsqueda de apoyo en ideas externas a la organización. Sin embargo, la puesta a prueba del marco de relaciones entre la ICA y la comunicación estratégica muestra que en las entidades consultadas los comunicadores no son los que proponen la creación de comunidades de colaboración con los diferentes grupos de stakeholders; tampoco se ocupan de liderar las negociaciones cuando se trata de materializar o comercializar ideas disruptivas, ni son los encargados de crear plataformas digitales para el trabajo en red con diferentes actores. Todas estas tareas son responsabilidad de las áreas especializadas en innovación y de dependencias que son punto de contacto entre las empresas y los grupos externos, como sucede entre las comunidades y las áreas de sostenibilidad, o los clientes y las áreas comerciales.

Hacia fuera, el acompañamiento que los comunicadores aportan en las diferentes etapas de los procesos de innovación es mayoritariamente de difusión, de distribución de informaciones, tanto en los medios institucionales como en la relación con periodistas, lo que indica una fuerte presencia de actividades outbound, relativas a lo que la empresa aporta a la sociedad. Las actividades inbound, mediante las cuales se capta información desde el entorno, se presentan en la relación entre los comunicado- 
res y sus proveedores de servicios, o entre los comunicadores y los medios informativos, de modo que así pueden ajustar sus trabajos a las demandas de estos dos grupos.

Los elementos del marco que se presentan con mayor frecuencia en la práctica de las entidades de la muestra son las relaciones con los proveedores y con las universidades, así como la existencia de incentivos estatales, sobre todo a través de Colciencias. La pertenencia a algunas redes, si bien en muchos casos depende del pago de una membresía, se establece para intercambiar conocimientos, actualizarse y hacer referenciación internacional. Un grupo de interés más bien ausente en las conversaciones sostenidas es la comunidad en general. Puede afirmarse que existe un mayor manejo o conocimiento de los procesos de la organización que inciden en la creación de una cultura innovadora, en lo que comprende la orientación estratégica y los comportamientos, y la existencia de áreas especializadas en gestionar la innovación, al ser los elementos del marco propositivo que se mencionan con mayor frecuencia en las conversaciones. Se da una presencia mayoritaria de públicos internos en los esfuerzos de los profesionales de la comunicación hacia la innovación, no obstante, la relación con los propios empleados es más bien tradicional y no hace parte de la ICA propiamente dicha.

En atención a los resultados, se podría aseverar que el entorno se expresa en las innovaciones que la organización adopta cuando estas son producto de su relación con los proveedores, con las universidades y con los medios de comunicación, que son los grupos de interés con los que se reconoce un proceso mucho más cercano a la manera como se concibe la ICA. No se perciben esfuerzos directos de las áreas y los responsables de comunicación por relacionarse con otros grupos que pueden hacer aportes sustanciales en materia de innovación, no solo para la entidad, sino para el propio proceso comunicativo, como los clientes y las comunidades. Se genera, por consiguiente, una tarea pendiente en lo que podrían ser procesos de construcción de sentido con públicos diferentes de los periodistas acerca de lo que se puede habilitar de manera conjunta entre la organización y la sociedad en general en materia de innovación. Este vacío ha sido explicado en estudios anteriores, en que los directivos de las empresas y otros miembros de las organizaciones no tienen muy claro el papel estratégico del profesio- 
nal de la comunicación (como sí perciben, muchas veces, a quienes se dedican a las ventas), y por ello solo lo contratan para generar contenidos y relacionarse con medios (Falkheimer et al., 2017; Preciado-Hoyos, NiviaFlórez \& Correales-Rivas, 2017). Otro asunto controversial viene siendo que se priorice la comunicación de las instituciones solo con los medios informativos, en un entorno en el que muchos más ciudadanos y grupos, y no solo los periodistas, se relacionan con las empresas de modo directo, a través de las redes sociales y otros soportes tecnológicos.

En estudios posteriores, convendría analizar, asimismo, otros puntos de contacto entre la organización y el entorno, pues cada vez más numerosos empleados se convierten en miembros activos en las redes de relación entre organización y los grupos de interés que pueden intervenir en optimizar procesos organizacionales como la innovación.

Aún quedan por resolver algunos vacíos que se presentan, en la literatura y en las compañías de la muestra aquí analizada, aunque se han encontrado algunas investigaciones previas en las que se analiza el trabajo en redes de innovación, a partir del registro conjunto de patentes (Yun, Jeong \& Park, 2016). En particular, en lo que compete al manejo de la marca, el reconocimiento de la autoría de las ideas que se comparten, la compensación de los stakeholders en las etapas de difusión y explotación de las innovaciones (Ettlinger, 2017; Zaggl, 2017) y cómo mantener la intensidad de la relación después de probar y aplicar las nuevas ideas.

La investigación futura sobre estas cuestiones, en Colombia y América Latina, deberá orientarse a realizar estudios sobre el papel que cumple la esfera pública en la construcción social de las organizaciones y a documentar aspectos que identifiquen la especificidad de las prácticas de los profesionales de la comunicación en las entidades de la región.

En vista de las dificultades para acceder a la información de las empresas, requerida en este estudio, es preciso insistir en que en este tipo de entidades se valore la investigación que realizan las universidades, desde la certeza de que es útil para ayudar a la mejora de las organizaciones y para la formación de los futuros profesionales. 


\section{Agradecimientos}

Al profesor David Hernández García, de la Facultad de Comunicaciones de la Universidad de Antioquia, por la aplicación de la entrevista realizada en Grupo Familia.

\section{Referencias}

Awazu, Y., Baloh, P., Desouza, K. C., Wecht, C. H., Kim, J. \& Jha, S. (2009). Information: Communication technologies open up innovation. Research-Technology Management, 52(1), 51-58. https://doi.org/ 10.1080/08956308.2009.11657548

Baldwin, C. \& Von Hippel, E. (2011). Modeling a paradigm shift: From producer innovation to user and open collaborative innovation. Organization Science, 22(6), 1399-1417. https://doi.org/10.1287/ orsc. 1100.0618

Bashir, N., Papamichail, K. N. \& Malik, K. (2017). Use of social media applications for supporting new product development processes in multinational corporations. Technological Forecasting and Social Change, 120, 176-183. https://doi.org/10.1016/j.techfore.2017.02.028

Bowen, S. A. (2008). A state of neglect: Public relations as 'corporate conscience'or ethics counsel. Journal of Public Relations Research, 20(3), 271-296. https://doi.org/10.1080/10627260801962749

Bvc.com. (s. f.).Listado de emisores. https://www.bvc.com.co/pps/tibco/portalbvc/Home/Empresas/Listado+de+Emisores?action=dummy

Cámara de Comercio de Medellín. (2017). Desempeño económico de Antioquia y perspectivas 2018. https://www.camaramedellin.com.co/ Portals/0/conozca_la_camara/informes-SIC/Informe-Economico-SIC2019.pdf 
Camargo, M. del P. (2013). Medellín, la ciudad más innovadora del mundo. Semana. https://www.semana.com/nacion/articulo/medellin-ciudad-mas-innovadora-del-mundo/334982-3

Carillo, K., Huff, S. \& Chawner, B. (2017). What makes a good contributor? Understanding contributor behavior within large Free/Open Source Software projects: A socialization perspective. The Journal of Strategic Information Systems, 26(4), 322-359. https://doi. org/10.1016/j.jsis.2017.03.001

Conrado, D.J., Karlsson, M. O., Romero, K., Sarr, C. \& Wilkins, J.J. (2017). Open innovation: Towards sharing of data, models and workflows. European Journal of Pharmaceutical Sciences, 109, S65-S71.https:// doi.org/10.1016/j.ejps.2017.06.035

Consejo Privado de Competitividad. (2018). El CPC. https://compite. com.co/el-cpc/

Consejo Privado de Competitividad. (2019). El CPC. https://compite. com.co/el-cpc/

Chesbrough, H. (2004). Managing open innovation. Research-Technology Management, 47(1), 23-26. https://doi.org/10.1080/08956308. 2004.11671604

Chesbrough, H. (2007). Why companies should have open business models. MIT Sloan Management Review, 48(2), 22-28. http://secure. com.sg/courses/ICI/Grab/Reading_Articles/L08_A02_Chesbrough.pdf

Chesbrough, H. \& Crowther, A. K. (2006). Beyond high tech: Early adopters of open innovation in other industries. Red Management, 36(3), 229-236. https://doi.org/10.1111/j.1467-9310.2006.00428.x

Dahlander, L. \& Gann, D. M. (2010). How open is innovation? Research Policy, 39(6), 699-709. https://doi.org/10.1016/j.respol.2010.01.013 
Dalmarco, G., Zawislak, P. A., Hulsink, W. \& Brambilla, F. (2015). How knowledge flows in university-industry relations: An overview from two economic sectors in Brazil. European Business Review, 27(2), 148-160. https://doi.org/10.1108/EBR-04-2013-0068

Dawson, B. K., Young, L., Tu, C. \& Chongyi, F. (2014). Co-innovation in networks of resources: A case study in the Chinese exhibition industry. Industrial Marketing Management, 43(3), 496-503. https: // doi.org/10.1016/j.indmarman.2013.12.017

Departamento Administrativo Nacional de Estadística. (2017, junio 29). Producto interno bruto departamental. Cuentas departamentales 2017: preliminar base 2015. https://www.dane.gov.co/ files/investigaciones/pib/departamentales/B_2015/pres dptal_2017preliminar.pdf

Dinero. (2018). Ranking de las empresas más innovadoras de Colombia en 2018. https://www.dinero.com/edicion-impresa/caratula/articulo/ranking-de-empresas-mas-innovadoras-de-colombiaen-2018/261002

Dinero. (2019). Medellín, pionera de la cuarta revolución industrial en la región. https://www.dinero.com/pais/articulo/centro-para-la-cuarta-revolucion-industrial-en-medellin/266221

Dittrich, K. \& Duysters, G. (2007). Networking as a means to strategy change: The case of open innovation in mobile telephony. Journal of Product Innovation Management, 24(6), 510-521. https://doi. org/10.1111/j.1540-5885.2007.00268.x

Dutta, S., Lanvin, B. \& Wunsch-Vincent, S. (Eds.). (2018). Global innovation index 2018: energizing the world with innovation. https://www. globalinnovationindex.org/gii-2018-report

El Colombiano. (2019, enero 23). Cuarta revolución industrial "made in" Medellín. http://www.elcolombiano.com/antioquia/medellin- 
centro-de-la-cuarta-revolucion-industrial-del-foro-economicomundial-HG10079970

Ettlinger, N. (2017). Open innovation and its discontents. Geoforum, 80, 61-71. https://doi.org/10.1016/j.geoforum.2017.01.011

Falkheimer, J., Heide, M., Nothhaft, H., von Platen, S., Simonsson, C., \& Andersson, R. (2017). Is Strategic Communication too important to be left to Communication Professionals? Managers' and coworkers' attitudes towards strategic communication and communication professionals. Public Relations Review, 43(1), 91-101. https://doi.org/10.1016/j.pubrev.2016.10.011

Ferdinand, J. P. \& Meyer, U. (2017). The social dynamics of heterogeneous innovation ecosystems: Effects of openess on community-firmas relations. Engineering Business Management, 9, 1-16. https://doi. org/10.1177/1847979017721617

Fernandes, S., Cesário, M. \& Barata, J. M. (2017). Ways to open innovation: Main agents and sources in the Portuguese case. Technology in Society, 51, 153-162.https://doi.org/10.1016/j.techsoc.2017.09.002

Fombrun, Ch. (1996). Reputation: Realizing value from the corporate image. Boston, EE. UU.: Harvard Business School Press.

Fuentes Navarro, R. (2005). Everett M. Rogers (1931-2004) y la investigación latinoamericana de la comunicación. Comunicación y Sociedad, 4, 93-125.http://www.scielo.org.mx/pdf/comso/n4/0188-252Xcomso-04-93.pdf

Füller, J., Matzler, K., Hutter, K. \& Hautz, J. (2012). Consumers' creative talent: Which characteristics qualify consumers for open innovation projetcs? An exploration of asymetrical effects. Creativity and Innovation Management, 21(3), 247-262. https://doi.org/10.1111/ j.1467-8691.2012.00650.x 
Gassmann, O. \& Enkel, E. (2004). Towards a theory of open innovation: Three core process archetypes. Trabajo presentado en R\&D Management Conference (RADMA), Lisboa, Portugal.

Hartley, J., Sørensen, E. \& Torfing, J. (2013). Collaborative innovation: A viable alternative to market competition and organizational entrepreneurship. Public Administration Review, 73(6), 821-830. https://doi.org/10.1111/puar.12136

Hoes, A. C., Beers, P. J. \& Van Mierlo, B. (2016). Communicating tensions among incumbents about system innovation in the Dutch dairy sector. Environmental Innovation and Societal Transitions, 21, 113122. https://doi.org/10.1016/j.eist.2016.04.005

Holtzhausen, D. \& Zerfass, A. (2015). Strategic communication: opportunities and challenges of the research area. En D. Holtzhausen y A. Zerfass (Eds.), The Routledge handbook of strategic communication. (pp. 27-41). Nueva York, EE. UU.: Routledge.

Ingenbleek, P. \& Dentoni, D. (2016). Learning from stakeholder pressure and embeddedness: The roles of absorptive capacity in the corporate social responsibility of Dutch Agribusinesses. Sustainability, 8(10). https://doi.org/10.3390/su8101026

Interacpedia. (2019). Empresas. https://www.interacpedia.com/

Islam, M., Miller, J. \& Park, H. D. (2017). But what will it cost me? How do private costs of participation affect open source software projects? Research Policy, 46(6), 1062-1070.https://doi.org/10.1016/j.respol.2017.05.005

Kratzer, J., Meissner, D. \& Roud, V. (2017). Open innovation and company culture: Internal openness makes the difference. Technological Forecasting and Social Change, 119, 128-138. https://doi. org/10.1016/j.techfore.2017.03.022 
Lee, S.M., Olson, D. L. \& Trimi, S. (2012). Co-innovation: Convergenomics, collaboration, and cocreation for organizational values. Management Decision, 50(5), 817-831.https://doi.org/10.1108/00251741211227528

Ley 1834/2017, de 23 de mayo, por medio de la cual se fomenta la economía creativa Ley Naranja El Congreso de Colombia. Diario Oficial, núm. 50.242 (2017).http://www.suin-juriscol.gov.co/viewDocument.asp?ruta $=$ Leyes $/ 30030647$

Lin, J. Y. (2017). Balancing industry collaboration and academic innovation: The contingent role of collaboration-specific attributes. Technological Forecasting and Social Change, 123, 216-228. https:// doi.org/10.1016/j.techfore.2016.03.016

Macnamara, J. (2015). Creating an "architecture of listening" in organizations: The basis of engagement, trust, healthy democracy, social equity, and business sustainability. Sídney, Australia: University of Technology Sydney.

Mahr, D. \& Lievens, A. (2012). Virtual lead user communities: Drivers of knowledge creation for innovation. Research Policy, 41(1), 167177. https://doi.org/10.1016/j.respol.2011.08.006

Marchi, G., Giachetti, C. \& De Gennaro, P. (2011). Extending lead-user theory to online brand communities: The case of the community Ducati. Technovation, 31(8), 350-361.https://doi.org/10.1016/j. technovation.2011.04.005

Prahalad, C. K. \& Ramaswamy, V. (2000). Co-opting customer competence. Harvard Business Review, 78(1), 79-90. https://pdfs.semanticscholar.org/b2a0/afdb89ef5cb64ad09cfa13d24851b3c9cf0a.pdf

Patsch, S. \& Zerfass, A. (2013). Co-innovation and communication: the case of SAP's global coinnovation lab network. En N. Pfeffermann, T. Minshall \& L. Mortara (Eds.), Strategy and communication for 
innovation. (pp. 397-414). Berlín, Alemania: Springer. https://doi. org/10.1007/978-3-642-41479-4_23

Patzer, M., Voegtlin, C. \& Scherer, A. G. (2018). The normative justification of integrative stakeholder engagement: A Habermasian view on responsible leadership. Business Ethics Quarterly, 28(3), 325354. https://doi.org/10.1017/beq.2017.33

Piller, F. \& West, J. (2013). Firms, users, and innovation: An interactive model of coupled open innovation. En H. Chesbrough, W. Vanhaverbeke \& J. West (Eds.), New frontiers in open innovation. (pp. 29-49). Oxford, RU: Oxford University Press, 2014.

Powell, W. W. (1990). Neither market nor hierarchy: Network forms of organization. En B. L. Staw \& L. Cummings (Eds.), Research in organizational behavior. (pp. 265-276). Greenwich, Londres: JAI Press.

Preciado-Hoyos, Á., Nivia-Flórez, A. M. \& Correales-Rivas, J. P. (2017). The strategic orientation of communications consulting firms in Colombia. Public Relations Review, 43(2), 406-416. https://doi. org/10.1016/j.pubrev.2017.02.003

Rankia. (2019, diciembre 12). ¿Qué es el Colcap? https://www.rankia.co/ blog/analisis-colcap/1578756-que-colcap

Restrepo Santamaría, N. (2011). Empresariado antioqueño y sociedad: influencia de las élites patronales de Antioquia en las políticas socioeconómicas colombianas. Medellín, Colombia: Universidad de Antioquia.

Safón, V. (2009). The moderating effect of the technological level of industry on the relationship between innovation and corporate reputation. International Entrepreneurship and Management Journal, 5(4), 515-526. https://doi.org/10.1007/s11365-009-0127-2 
Sagaris, L. (2014). Citizen participation for sustainable transport: The case of "Living City" in Santiago, Chile (1997-2012). Journal of Transport Geopraphy, 41, 74-83. https://doi.org/10.1016/j.jtrangeo.2014.08.011

Savič, I. (2016). Mediatization of companies as a factor of their communication power and the new role of public relations. Public Relations Review, 42(4), 607-615. https://doi.org/10.1016/j.pubrev.2016.03.017

Scandura, A. (2016). University-industry collaboration and firms' R\&D effort. Research Policy, 45(9), 1907-1922. https://doi. org/10.1016/j.respol.2016.06.009

Schröder, A. \& Hölzle, K. (2010). Virtual communities for innovation: Influence factors and impact on company innovation. Creativity and Innovation Management, 19(3), 257-268. https://doi. org/10.1111/j.1467-8691.2010.00567.x

Schumpeter, J. A. (1934). The theory of economic development. Cambridge, EE. UU.: Harvard University Press.

Scuotto, V., Del Giudice, M., Della Peruta, M. R. \& Tarba, S. (2017). The performance implications of leveraging internal innovation through social media networks: An empirical verification of the smart fashion industry. Technological Forecasting and Social Change, 120, 184194. https://doi.org/10.1016/j.techfore.2017.03.021

Shinkevich, A. I., Galimulina, F. F., Moiseyev, V. O., Avilova, V. V., Kuramshina, K. S., Ishmuradova, I. I. ... Grankina, V. L. (2016). Features of integrative relations between science, state and industry in Russia and abroad. International Review of Management and Marketing, 6(2S), 142-148. https://dspace.kpfu.ru/xmlui/handle/ net/145174 
Tecnova. (2019). Conócenos. http://www.tecnnova.org/tecnnova/

Tossavainen, T., Shiramatsu, S., Ozono, T. \& Shintani, T. (2016). A linked open data based system utilizing structured open innovation process for addressing collaboratively public concerns in regional societies. Applied Intelligence, 44(1), 196-207. https://doi. org/10.1007/s10489-015-0704-8

Urgal, B., Quintás, M. Á. y Arévalo Tomé, R. (2011). Conocimiento tecnológico, capacidad de innovación y desempeño innovador: el rol moderador del ambiente interno de la empresa. Cuadernos de Economía y Dirección de la Empresa, 14(1), 53-66. https://doi. org/10.1016/j.cede.2011.01.004

Verhoeven, P., Zerfass, A. \& Tench, R. (2011). Strategic orientation of communication professionals in Europe. International Journal of Strategic Communication, 5(2), 95-117. https://doi.org/10.1080/15 53118X.2011.561080

Von Hippel, X. (1988). The sources of innovation. Nueva York, EE. UU.: Oxford University Press.

Von Platen, S. (2015). The communication consultant: An important translator for communication management. Journal of Communication Management, 19(2), 150-166, https://doi.org/10.1108/JCOM06-2013-0049

Von Platen, S. (2016). Struggling with new media and old expertise: Reconstructing the professional role of communication consultancy. International Journal of Strategic Communication, 10(5), 353-367. https://doi.org/10.1080/1553118X.2016.1204612

Wakefield, R. \& Wakefield, K. (2016). Social media network behavior: A study of user passion and affect. The Journal of Strategic Information Systems, 25(2), 140-156. https://doi.org/10.1016/j.jsis.2016.04.001 
West, J. \& Bogers, M. (2017). Open innovation: Current status and research opportunities. Innovation, 19(1), 43-50. https://ssrn.com/ abstract $=2864393$

West, J. \& Gallager, S. (2006). Patterns of open innovation in open source software. En H. Chesbrough, W. Vanhaverbeke y J. West (Eds.), Open innovation: Researching a new paradigm. (pp. 1-46). Boston, EE. UU.: Oxford University Press.

Yun, J.J., Jeong, E. \& Park, J. (2016). Network analysis of open innovation. Sustainability, 8(8), 729. https://doi.org/10.3390/su8080729

Zaggl, M. A. (2017). Manipulation of explicit reputation in innovation and knowledge exchange communities: The example of referencing in science. Research Policy, 46(5), 970-983. https://doi. org/10.1016/j.respol.2017.02.009 